\title{
THE $P_{2}^{1}$ MARGOLIS HOMOLOGY OF CONNECTIVE TOPOLOGICAL MODULAR FORMS
}

\author{
PRASIT BHATTACHARYA, IRINA BOBKOVA, AND BRIAN THOMAS
}

\begin{abstract}
The element $\mathrm{P}_{2}^{1}$ of the mod 2 Steenrod algebra $\mathcal{A}$ has the property $\left(\mathrm{P}_{2}^{1}\right)^{2}=0$. This property allows one to view $\mathrm{P}_{2}^{1}$ as a differential on $H_{*}\left(X, \mathbb{F}_{2}\right)$ for any spectrum $X$. Homology with respect to this differential, $\mathcal{M}\left(X, \mathrm{P}_{2}^{1}\right)$, is called the $\mathrm{P}_{2}^{1}$ Margolis homology of $X$. In this paper we give a complete calculation of the $\mathrm{P}_{2}^{1}$ Margolis homology of the 2-local spectrum of topological modular forms tmf and identify its $\mathbb{F}_{2}$ basis via an iterated algorithm. We apply the same techniques to calculate $\mathrm{P}_{2}^{1}$ Margolis homology for any smash power of $t m f$.
\end{abstract}

1. Introduction

2. Action of $P_{2}^{1}$ and the length spectral sequence 6

3. The reduced length 12

4. $P_{2}^{1}$ Margolis homology of $t m f^{\wedge r}$ and $B\left(\mathbb{Z} / 2^{\times n}\right)_{+} \quad 20$

References 23

Contents

Convention. Throughout this paper we work in the stable homotopy category of spectra localized at the prime 2 .

\section{INTRODUCTION}

The connective $E_{\infty}$ ring spectrum of topological modular forms tmf has played a vital role in computational aspects of chromatic homotopy theory over the last two decades [Goe10], [DFHH14]. It is essential for detecting information about the chromatic height 2 , and it has the rare quality of having rich Hurewicz image. There is a $K(2)$-local equivalence [HM14]

$$
L_{K(2)} t m f \simeq E_{2}^{h G_{48}}
$$

where $E_{2}$ is the second Morava $E$-theory at $p=2$ and $G_{48}$ is the maximal finite subgroup of the Morava stabilizer group $\mathbb{G}_{2}$. The spectrum $E_{2}^{h G_{48}}$ can be used to build the $K(2)$-local sphere spectrum (see [BG18]). The homotopy groups of $t m f$ approximate both the stable homotopy groups of spheres and the ring of integral modular forms. In many senses, $t m f$ is the chromatic height 2 analogue of connective real $K$-theory $k o$. Further, the homotopy groups of $t m f$ are completely known [Bau08].

Let us now recall the definition of the element $\mathrm{P}_{2}^{1} \in \mathcal{A}$. Milnor described the mod 2 dual Steenrod algebra $\mathcal{A}_{*}$ as the graded polynomial algebra [Mil58, App. 1]

$$
\mathcal{A}_{*} \cong \mathbb{F}_{2}\left[\xi_{1}, \xi_{2}, \xi_{3}, \ldots\right] \text {, }
$$


where $\left|\xi_{i}\right|=2^{i}-1$. The Steenrod algebra $\mathcal{A}$ has an $\mathbb{F}_{2}$-basis dual to the monomial basis of $\mathcal{A}_{*}$. The elements of the $\mathbb{F}_{2}$-basis of $\mathcal{A}$ which are dual to $\xi_{t}^{2^{s}}$ are denoted by $\mathrm{P}_{t}^{s}$, and the elements $\mathrm{P}_{t}^{0}$ are denoted by $\mathrm{Q}_{t-1}$. When $s<t$, the elements $\mathrm{P}_{t}^{s}$ are exterior power generators, i.e. $\left(\mathrm{P}_{t}^{s}\right)^{2}=0$. Thus, any left $\mathcal{A}$-module $K$ can be regarded as a complex with differential given by the left multiplication by $\mathrm{P}_{t}^{s}$ (for $s<t$ ). This leads to the following definition.

Definition 1.1 ([Mar83]). Let $K$ be any left $\mathcal{A}$-module and $0 \leq s<t$. Let

$$
{ }^{L} \mathcal{P}_{t}^{s}: K \longrightarrow K
$$

denote the left action by $\mathrm{P}_{t}^{s}$. The left $\mathrm{P}_{t}^{s}$ Margolis homology group of $K, \mathcal{M}^{L}\left(K, \mathrm{P}_{t}^{s}\right)$, is defined as

$$
\mathcal{M}^{L}\left(K, \mathrm{P}_{t}^{s}\right):=\frac{\operatorname{Ker}^{L} \mathcal{P}_{t}^{s}: K \rightarrow K}{\operatorname{Im}^{L} \mathcal{P}_{t}^{s}: K \rightarrow K} .
$$

For a right $\mathcal{A}$-module $K$, one can similarly define the right $\mathrm{P}_{t}^{s}$ Margolis homology group of $K$ as

$$
\mathcal{M}^{R}\left(K, \mathrm{P}_{t}^{s}\right):=\frac{\operatorname{Ker}^{R} \mathcal{P}_{t}^{s}: K \rightarrow K}{\operatorname{Im}^{R} \mathcal{P}_{t}^{s}: K \rightarrow K}
$$

where ${ }^{R} \mathcal{P}_{t}^{s}$ is the right action by $\mathrm{P}_{t}^{s}$ on $K$.

Notation 1.2. For a spectrum $X, \mathcal{M}\left(X, \mathrm{P}_{t}^{s}\right)$ will denote $\mathcal{M}^{L}\left(H^{*}(X), \mathrm{P}_{t}^{s}\right)$ or equivalently $\mathcal{M}^{R}\left(H_{*}(X), \mathrm{P}_{t}^{s}\right)$.

Computations of Margolis homology underly many essential computations in homotopy theory. For example, Adams work on $B P\langle 1\rangle$ cooperations [Ada74] relies on the computations of $\mathcal{M}\left(B P\langle 1\rangle, \mathrm{Q}_{i}\right)$ for $i=0,1$. Calculations like $\mathcal{M}\left(b o, \mathrm{Q}_{i}\right)$ for $i=0,1$ are essential ingredients in the work of Mahowald on bo-resolutions [Mah81]. More recently, Culver described $B P\langle 2\rangle$ resolutions [Cul] by understanding $\mathcal{M}\left(B P\langle 2\rangle, \mathrm{Q}_{i}\right)$ for $i=0,1,2$. Computation of $\mathcal{M}\left(t m f^{\wedge n}, \mathrm{Q}_{2}\right)$ is an essential ingredient in $\left[\mathrm{BBB}^{+} \mathrm{b}\right]$.

The element $Q_{i}$ is primitive for all $i \in \mathbb{N}$. In other words, the comultiplication map $\Delta$ on $\mathcal{A}$ sends $Q_{i}$ to

$$
\Delta\left(Q_{i}\right)=Q_{i} \otimes 1+1 \otimes Q_{i} .
$$

Consequently, $\mathrm{Q}_{i}$ acts on $H_{*}(X)$ as a derivation, namely it follows the Leibniz rule

$$
\mathrm{Q}_{i}(x y)=\mathrm{Q}_{i}(x) \cdot y+x \cdot \mathrm{Q}_{i}(y),
$$

whenever $X$ is a ring spectrum. The Leibniz rule implies the Künneth isomorphism [Mar83, Proposition 17, pg 343]

$$
\mathcal{M}\left(X \otimes Y, \mathrm{Q}_{i}\right) \cong \mathcal{M}\left(X, \mathrm{Q}_{i}\right) \otimes \mathcal{M}\left(Y, \mathrm{Q}_{i}\right)
$$

and hence, $\mathcal{M}\left(X, \mathrm{Q}_{i}\right)$ is an $\mathbb{F}_{2}$ algebra whenever $X$ is a ring spectrum. As a result, computation of $Q_{i}$ Margolis homology and its description is often fairly straightforward.

On the other hand, for $s>0, \mathrm{P}_{t}^{s}$ is not a primitive element of $\mathcal{A}$. In particular,

$$
\Delta\left(P_{2}^{1}\right)=P_{2}^{1} \otimes 1+Q_{1} \otimes Q_{1}+1 \otimes P_{2}^{1}
$$


and its action on $H_{*}(X)$ for a ring spectrum $X$, does not follow the Leibniz rule. Instead, we have

$$
\mathrm{P}_{2}^{1}(x y)=\mathrm{P}_{2}^{1}(x) y+\mathrm{Q}_{1}(x) \mathrm{Q}_{1}(y)+x \mathrm{P}_{2}^{1}(y) .
$$

As a result, the product of two $\mathrm{P}_{2}^{1}$ cycles may not necessarily be a $\mathrm{P}_{2}^{1}$ cycle, hence $\mathcal{M}\left(X, \mathrm{P}_{2}^{1}\right)$ may not admit any multiplicative structure even if $X$ is a ring spectrum. This is the main reason why the $\mathrm{P}_{2}^{1}$ Margolis homology calculations are significantly more complicated.

Let us now consider the spectrum tmf. It is well-known ([HM14], [Mat16]) that

$$
H_{*}\left(t m f ; \mathbb{F}_{2}\right) \cong \mathbb{F}_{2}\left[\zeta_{1}^{8}, \zeta_{2}^{4}, \zeta_{3}^{2}, \zeta_{4}, \zeta_{5}, \ldots\right] \subset \mathcal{A}_{*}
$$

is a subalgebra of $\mathcal{A}_{*}$. Here the elements $\zeta_{i}$ are the images of $\xi_{i}$ under the antipode of the Hopf algebra $\mathcal{A}_{*}$ (see Section 2). The right action of $\mathrm{Q}_{i}$ is given by the formula (see [Cul, §2] for details)

$$
\mathrm{Q}_{i}\left(\zeta_{n}\right)=\zeta_{n-i-1}^{2^{i+1}}
$$

Then, since the $Q_{i}$ are derivations, it can be easily seen that

$$
\begin{gathered}
\mathcal{M}\left(t m f, \mathrm{Q}_{0}\right)=\mathbb{F}_{2}\left[\zeta_{1}^{8}, \zeta_{2}^{4}\right] \\
\mathcal{M}\left(t m f, \mathrm{Q}_{1}\right)=\frac{\mathbb{F}_{2}\left[\zeta_{1}^{8}, \zeta_{3}^{2}, \zeta_{4}^{2}, \ldots\right]}{\left\langle\zeta_{3}^{4}, \zeta_{4}^{4}, \ldots\right\rangle} \\
\mathcal{M}\left(t m f, \mathrm{Q}_{2}\right)=\frac{\mathbb{F}_{2}\left[\zeta_{2}^{4}, \zeta_{3}^{2}, \zeta_{4}^{2}, \ldots\right]}{\left\langle\zeta_{2}^{8}, \zeta_{3}^{8}, \zeta_{4}^{8}, \ldots\right\rangle} .
\end{gathered}
$$

In this paper, we give a complete calculation of $\mathcal{M}\left(t m f^{\wedge r}, \mathrm{P}_{2}^{1}\right)$ for arbitrary $r \geq 1$. In fact, the calculation for $r>1$ follows from the case $r=1$, because after forgetting the internal grading one can construct a non-canonical isomorphism (see Section 4)

$$
\mathcal{M}\left(t m f^{\wedge r}, \mathrm{P}_{2}^{1}\right) \cong \mathcal{M}\left(t m f, \mathrm{P}_{2}^{1}\right) .
$$

For the case $r=1$, we give an iterated algorithm (see Definition 3.16) that constructs an $\mathbb{F}_{2}$-basis of $\mathcal{M}\left(t m f, \mathrm{P}_{2}^{1}\right)$. We give a complete description of $\mathcal{M}\left(t m f, \mathrm{P}_{2}^{1}\right)$ in Theorem 3.18 which is the main result of this paper. Although $\mathcal{M}\left(\operatorname{tmf}, \mathrm{P}_{2}^{1}\right)$ is not an algebra, we notice that $\mathcal{M}\left(t m f, \mathrm{P}_{2}^{1}\right)$ is a module over an infinitely generated exterior algebra $\mathcal{S}$ (see Lemma 3.1 for a description of $\mathcal{S}$ ). Theorem 3.18 also describes $\mathcal{M}\left(t m f, \mathrm{P}_{2}^{1}\right)$ as an $\mathcal{S}$-module.

The key tool we use is the length spectral sequence (2.14), which we define in Section 2. The length spectral sequence admits a $d_{0}$ differential and a $d_{2}$ differential and collapses at the $E_{3}$ page. The Leibniz rule does hold for the $d_{0}$, but not for $d_{2}$. In order to work around this issue, we notice that the $E_{2}$ page admits an action of $\mathcal{S}$ (i.e. $d_{2}$ are $\mathcal{S}$ linear) and we use it to simplify the computation of $E_{\infty}=E_{3}$.

We also notice that almost identical calculations lead to a complete description of $\mathcal{M}\left(\left(B \mathbb{Z} / 2^{\times n}\right)_{+}, \mathrm{P}_{2}^{1}\right)$. The methods developed in this paper can be considered as a blueprint for computations of $P_{t}^{1}$ Margolis homology of a variety of other $\mathcal{A}$-modules.

Our calculations of $\mathcal{M}\left(t m f^{\wedge r}, \mathrm{P}_{2}^{1}\right)$ have many applications, as the spectrum tmf has a wide range of applications, particularly in chromatic homotopy theory. First 
note that the cohomology of $\operatorname{tmf}$, as a module over the Steenrod algebra $\mathcal{A}$, is isomorphic to (see [HM14], [Mat16])

$$
H^{*}\left(t m f ; \mathbb{F}_{2}\right) \cong \mathcal{A} / / \mathcal{A}(2)
$$

where $\mathcal{A}(2)$ is the subalgebra of $\mathcal{A}$ generated by $\mathrm{Sq}^{1}, \mathrm{Sq}^{2}$ and $\mathrm{Sq}^{4}$. This, and a change of rings isomorphism, imply that the $E_{2}$ page of the Adams spectral sequence converging to $t m f_{*} X$ (for a spectrum $X$ ) is

$$
E_{2}^{s, t}:=\operatorname{Ext}_{\mathcal{A}(2)}^{s, t}\left(H^{*}(X), \mathbb{F}_{2}\right) .
$$

One can detect infinite families in the $E_{2}$ page via the map

$$
q: \operatorname{Ext}_{\mathcal{A}(2)}^{s, t}\left(H^{*}(X), \mathbb{F}_{2}\right) \longrightarrow \operatorname{Ext}_{\Lambda\left(\mathrm{P}_{2}^{1}\right)}^{s, t}\left(H^{*}(X), \mathbb{F}_{2}\right) .
$$

The codomain of $q$ can be understood by calculating $\mathcal{M}\left(X, \mathrm{P}_{2}^{1}\right)$. Note that

$$
\operatorname{Ext}_{\Lambda\left(\mathrm{P}_{2}^{1}\right)}^{s, t}\left(\mathbb{F}_{2}, \mathbb{F}_{2}\right) \cong \mathbb{F}_{2}\left[h_{2,1}\right]
$$

where $\left|h_{2,1}\right|=(1,6)$ and

$$
\mathbb{F}_{2}\left[h_{2,1}\right] \otimes \mathcal{M}\left(X, \mathrm{P}_{2}^{1}\right) \subset \operatorname{Ext}_{\Lambda\left(\mathrm{P}_{2}^{1}\right)}^{s, t}\left(H^{*}(X), \mathbb{F}_{2}\right)
$$

accounts for all the elements with positive $s$ filtration. This shows that the knowledge of $\mathcal{M}\left(X, \mathrm{P}_{2}^{1}\right)$ is crucial in detecting patterns in the $E_{2}$-page of (1.9).

Motivation I - Towards homotopy groups of $K(2)$-local sphere. Computation of the homotopy groups of $L_{K(n)} S^{0}$ - the sphere spectrum localized with respect to Morava $K$-theories $K(n)$ at various primes $p$ and heights $n$ - is the central question of chromatic homotopy theory. It is sometimes easier to compute $\pi_{*} L_{K(n)} X$ for finite complexes other than the sphere, although very little data like this is known at $n=p=2$ anyway. Recently, Bhattacharya and Egger introduced a family of finite spectra $Z$ [BEa], and $\pi_{*} L_{K(2)} Z$ has been computed [BBB ${ }^{+} \mathrm{b}, \mathrm{BEb}$ ], the first example of a finite complex at $p=2$ whose $K(2)$-local homotopy groups are completely determined. The finite complex $Z$ can be constructed from the sphere spectrum, by a succession of cofiber sequences of self-maps (see [BEa], the last one of which is

$$
\Sigma^{5} A_{1} \wedge C \nu \stackrel{w}{\longrightarrow} A_{1} \wedge C \nu \longrightarrow Z .
$$

In a quest to leverage the knowledge of $\pi_{*} L_{K(2)} Z$ to $\pi_{*} L_{K(2)} S^{0}$, one must first attempt to compute the $K(2)$-local homotopy groups of $A_{1} \wedge C \nu$. Very briefly, our strategy is to use the $v_{2}$-local $t m f$-based Adams spectral sequence

$$
E_{1}^{r, t}=v_{2}^{-1} \pi_{t}\left(t m f \wedge \overline{t m f}^{\wedge r} \wedge A_{1} \wedge C \nu\right) \Longrightarrow \pi_{t-r}\left(L_{K(2)} A_{1} \wedge C \nu\right)
$$

and compare it with that of $Z$. One can identify the $E_{1}$-page of the above spectral sequence using the classical Adams spectral sequence

$$
E_{2}^{s, t}=\operatorname{Ext}_{\mathcal{A}}^{s, t}\left(H^{*}\left(t m f \wedge \overline{t m f}^{\wedge r} \wedge A_{1} \wedge C \nu\right), \mathbb{F}_{2}\right) \Rightarrow \pi_{t-s}\left(t m f \wedge \overline{t m f}^{\wedge r} \wedge A_{1} \wedge C \nu\right) .
$$

Because of (1.8) and the fact that $H^{*}\left(A_{1} \wedge C \nu\right) \cong \mathcal{A}(2) / / \Lambda\left(\mathrm{Q}_{2}, \mathrm{P}_{2}^{1}\right)$, and the change of rings isomorphism, the $E_{2}$-page of the spectral sequence (1.10) has the form

$$
\operatorname{Ext}_{\Lambda\left(\mathrm{Q}_{2}, \mathrm{P}_{2}^{1}\right)}^{s,}\left(H^{*}\left(\overline{\operatorname{tmf}}^{\wedge r}\right), \mathbb{F}_{2}\right)
$$

Hence, computation of $\mathcal{M}\left(t m f^{\wedge r}, \mathrm{P}_{2}^{1}\right)$ is essential for understanding the $E_{2}$-page of (1.10). 
Motivation II - tmf resolution of the sphere spectrum. The connective spectrum $b o$ is not a flat ring spectrum, hence the $E_{2}$ page of the $b o$-based Adams spectral sequence does not have a straightforward expression like the classical Adams spectral sequence. However, Lellmann and Mahowald [LM87] were able to calculate the $d_{1}$ differentials (also see $\left[\mathrm{BBB}^{+} \mathrm{a}\right]$ ) and gave a description of the " $v_{1}$-periodic part" of the $E_{2}$-page. They identified the free Eilenberg-MacLane summand of $b o^{\wedge r}$. To identify this free summand one needs to identify the $\mathcal{A}(1)$ free summand of

$$
H^{*}\left(b o^{\wedge r}\right) \cong \mathcal{A} / / \mathcal{A}(1)^{\otimes r} .
$$

This can be done by calculating $\mathcal{M}\left(b o^{\wedge r}, \mathrm{Q}_{0}\right)$ and $\mathcal{M}\left(b o^{\wedge r}, \mathrm{Q}_{1}\right)$ and using the following theorem due to Margolis.

Theorem 1.11 ([Mar83, Chapter 19, Theorem 6]). An $\mathcal{A}(n)$-module $K$ is free if and only if $\mathcal{M}\left(K, \mathrm{P}_{t}^{s}\right)=0$ whenever $s+t \leq n+1$ with $s<t$.

To emulate the strategy of Lellmann and Mahowald to understand the tmf-based Adams spectral sequence for $S^{0}$ one needs to first identify the $\mathcal{A}(2)$-free part of

$$
H^{*}\left(t m f^{\wedge r}\right) \cong(\mathcal{A} / / \mathcal{A}(2))^{\otimes r} .
$$

Potentially, this can be identified using the knowledge of $\mathcal{M}\left(t m f^{\wedge r}, \mathrm{Q}_{i}\right)$ for $i=0,1,2$ and $\mathcal{M}\left(t m f^{\wedge r}, \mathrm{P}_{2}^{1}\right)$, along with Theorem 1.11.

Motivation III - Infinite loop space of tmf. There are $\mathcal{A}$-modules $J(k)$, called Brown-Gitler modules [BG73], which assemble into a doubly graded $\mathcal{A}$-algebra, denoted here by $J(*)^{*}$. Moreover, there is an $\mathcal{A}$-module isomorphism $J(*)^{*} \cong$ $\mathbb{F}_{2}\left[x_{1}, x_{2}, \ldots\right]$ where $x_{i} \in J\left(2^{i}\right)^{1}$ and the left $\mathcal{A}$ action on $J(*)^{*}$ is [Sch94]

$$
\mathrm{Sq}\left(x_{i}\right)=x_{i}+x_{i-1}^{2} \text {. }
$$

In fact, $J(k)^{*}$ can be thought of as inheriting this action by virtue of being a subobject of $\mathcal{A}$. Because of this, minor modifications to methods of this paper apply to the calculation of $\mathcal{M}\left(J(k), \mathrm{P}_{2}^{1}\right)$. By [KM13] there is a spectral sequence, obtained by studying Goodwillie towers, relating the knowledge of $H_{*}\left(t m f ; \mathbb{F}_{2}\right)$ to that of $H_{*}\left(\Omega^{\infty} t m f ; \mathbb{F}_{2}\right)$ (also see [HM16] which provides a spectral sequence relating the cohomology of tmf to the cohomology of its infinite loop-space $\left.H^{*}\left(\Omega^{\infty} t m f ; \mathbb{F}_{2}\right)\right)$. Roughly speaking, this relies on computing certain derived functors, usually labeled $\Omega_{s}^{\infty}$, in the category of unstable modules over $\mathcal{A}$. It turns out that there is an isomorphism (see [Goe86] or [HK00])

$$
\Omega_{s}^{\infty} \Sigma^{-t}(\mathcal{A} / / \mathcal{A}(2))_{*} \cong \operatorname{Ext}_{\mathcal{A}(2)}^{s, t}\left(\mathbb{F}_{2}, J(*)\right),
$$

so that these computations require an understanding of the $J(k)$ as modules over $\mathcal{A}(2)$, the hardest part of which is understanding how $\mathrm{P}_{2}^{1}$ acts.

Organization of the paper. In Section 2, we recall some facts about the Steenrod algebra and its dual. We introduce the spectral sequence (2.14), which computes the $\mathrm{P}_{2}^{1}$ Margolis homology of tmf, and discuss the $d_{0}$ differentials in it.

In Section 3, we compute the $E_{3}=E_{\infty}$ page of the spectral sequence (2.14). We do that by introducing building blocks $M_{J}$ and computing $\mathcal{M}\left(M_{J}, \mathrm{P}_{2}^{1}\right)$. Then we establish the relationship between $\mathcal{M}\left(t m f, \mathrm{P}_{2}^{1}\right)$ and $\mathcal{M}\left(M_{J}, \mathrm{P}_{2}^{1}\right)$ in Theorem 3.18. 
In Section 4, we show how to apply the same methods to calculate $\mathrm{P}_{2}^{1}$ Margolis homology for $t m f^{\wedge r}$ and $\left(B \mathbb{Z} / 2^{\times k}\right)_{+}$. Theorem 3.18 essentially gives complete answer in these cases.

Acknowledgments. The authors are indebted to Nicolas Ricka for many insightful conversations. We are grateful to Nick Kuhn, Haynes Miller and John Rognes for their help and comments. We would like to thank the anonymous referee for many helpful comments and suggestions for improvement. This material is based upon work supported by the National Science Foundation Grant DMS-1440140, while the second author was in residence at the MSRI during the Spring 2019 semester, and Grants DMS-1638352 and DMS-2005627.

\section{Action of $P_{2}^{1}$ And the Length spectral Sequence}

The dual Steenrod algebra $\mathcal{A}_{*}=\pi_{*}\left(H \mathbb{F}_{2} \wedge H \mathbb{F}_{2}\right)$ has the structure of a graded commutative algebra which Milnor [Mil58] showed to be a polynomial algebra

$$
\mathcal{A}_{*} \cong \mathbb{F}_{2}\left[\xi_{1}, \xi_{2}, \xi_{3}, \ldots\right]
$$

where $\left|\xi_{i}\right|=2^{i}-1$. Milnor defined $\mathrm{Sq}\left(r_{1}, r_{2}, \ldots\right) \in \mathcal{A}$ as the dual of $\xi_{1}^{r_{1}} \xi_{2}^{r_{2}} \ldots$ and showed that they form an $\mathbb{F}_{2}$ basis of the Steenrod algebra $\mathcal{A}$, known as the Milnor basis. The $\mathrm{P}_{t}^{s}$ elements are defined as

$$
\mathrm{P}_{t}^{s}=\mathrm{Sq}\left(r_{1}, \ldots\right), \text { where } r_{i}= \begin{cases}0, & i \neq t \\ 2^{s}, & i=t .\end{cases}
$$

The action of an element $a \in \mathcal{A}$ on an $\mathcal{A}$-algebra follows the product rule given by the Cartan formula, i.e.

$$
a(x \cdot y)=\Sigma_{i} a_{i}^{\prime}(x) \cdot a_{i}^{\prime \prime}(y) .
$$

where $\Delta(a)=\Sigma_{i} a_{i}^{\prime} \otimes a_{i}^{\prime \prime}$ is the comultiplication in the Hopf algebra $\mathcal{A}$.

Remark 2.1. We would like to note that standard commonly used notation for the generators of the dual Steenrod algebra at $p=2$ differs from the notation in the original paper [Mil58], and we are grateful to John Rognes for explaining this to us. In [Mil58, Appendix 1], Milnor denotes the polynomial generators of the dual Steenod algebra at $p=2$ by $\zeta_{i}$, so that $\mathcal{A}_{*} \cong \mathbb{F}_{2}\left[\zeta_{1}, \zeta_{2}, \ldots\right]$ and defines $\mathrm{Sq}\left(r_{1}, r_{2}, \ldots\right)$ as dual to the element $\zeta_{1}^{r_{1}} \zeta_{2}^{r_{2}} \ldots$. It has since become standard in the literature [MT68, Ada74, Mar83] to use a different notation and to denote the polynomial generators which were denoted by $\zeta_{i}$ in [Mil58, Appendix 1] by $\xi_{i}$, in order to match the notation for the odd primary Steenrod algebra. Hence in current standard notation $\mathrm{Sq}\left(r_{1}, r_{2}, \ldots\right)$ is dual to $\xi_{1}^{r_{1}} \xi_{2}^{r_{2}} \cdots$. The symbol $\zeta_{i}$ is now usually used to denote the image of $\xi_{i}$ under the antipode of the Hopf algebra $\chi: \mathcal{A}_{*} \rightarrow \mathcal{A}_{*}$, induced by the 'flip map' on $H \mathbb{F}_{2} \wedge H \mathbb{F}_{2}$. The elements $\zeta_{i}=\chi\left(\xi_{i}\right)$ can be computed recursively using the formula $\sum_{i+j=k} \xi_{i}^{2^{j}} \chi\left(\xi_{j}\right)=0$, together with the assumption that $\xi_{0}=1$ and $\xi_{i}=0$ when $i<0$.

The homology of tmf is the subalgebra of $\mathcal{A}_{*}$ ([HM14], [Mat16, Theorem 5.13])

$$
\mathfrak{T}:=H_{*}\left(t m f ; \mathbb{F}_{2}\right) \cong(\mathcal{A} / / \mathcal{A}(2))_{*}=\mathbb{F}_{2}\left[\zeta_{1}^{8}, \zeta_{2}^{4}, \zeta_{3}^{2}, \zeta_{4}, \zeta_{5}, \ldots\right] .
$$

Thus the action of $\mathcal{A}$ on $\mathfrak{T}$ is simply the restriction of the action of $\mathcal{A}$ on $\mathcal{A}_{*}$. 
The right action of $\mathcal{A}$ on $\mathcal{A}_{*}$ is determined by the action of the total squaring operation $\mathrm{Sq}=1+\sum_{i>0} \mathrm{Sq}^{i}$ [Pea14, Lemma 3.6]

$$
\left(\zeta_{i}\right) \mathrm{Sq}=\zeta_{i}+\zeta_{i-1}^{2}+\zeta_{i-2}^{4}+\cdots+\zeta_{1}^{2^{i-1}}+1
$$

which is a ring homomorphism.

Remark 2.4 (Action of the total squaring operation). There are multiple ways to define the action of $\mathcal{A}$ on $\mathcal{A}_{*}$. While we will be using the action defined by (2.3), we would like to collect other commonly used actions here. By [Mah81], the right and left actions of Sq on $\xi_{i}$ are given by the formulas

$$
\begin{aligned}
& \mathrm{Sq}\left(\xi_{i}\right)=\xi_{i}+\xi_{i-1}^{2} \\
& \left(\xi_{i}\right) \mathrm{Sq}=\xi_{i}+\xi_{i-1},
\end{aligned}
$$

while the left action on $\zeta_{i}$ is

$$
\mathrm{Sq}\left(\zeta_{i}\right)=\zeta_{i}+\zeta_{i-1}+\cdots+\zeta_{1}+1 .
$$

From these formulas we can derive

$$
\begin{aligned}
Q_{i-1}\left(\xi_{n}\right) & =\xi_{n-i}^{2^{i}} \\
\left(\zeta_{n}\right) Q_{i-1} & =\zeta_{n-i}^{2^{i}} ;
\end{aligned}
$$

the second equation can also be found in [Cul].

Important Notation 2.5. Since we only work with the right action of Sq in this paper, we will write $a(x)$ to denote the right action of $a \in \mathcal{A}$ on $x \in H_{*}($ tmf $)$ for the rest of the paper. Thus, from now on

$$
a(x):=(x) a .
$$

We now focus on the action of $\mathrm{P}_{2}^{1}=\mathrm{Sq}(0,2)=\mathrm{Sq}^{2} \mathrm{Sq}^{4}+\mathrm{Sq}^{4} \mathrm{Sq}^{2}$ on $\mathfrak{T}$. From (2.3), one can easily see that $\mathrm{Sq}^{2 i}$ acts trivially on $\zeta_{n}$, when $i>0$ and $n \neq 1$. It follows immediately that

$$
\mathrm{P}_{2}^{1}\left(\zeta_{i}\right)=0
$$

Beware! This does not mean that $\mathrm{P}_{2}^{1}\left(\zeta_{i} \zeta_{j}\right)=0$, as the Leibniz rule does not hold. Since $\Delta\left(\mathrm{P}_{2}^{1}\right)=\mathrm{P}_{2}^{1} \otimes 1+\mathrm{Q}_{1} \otimes \mathrm{Q}_{1}+1 \otimes \mathrm{P}_{2}^{1}$, we obtain the product formula

$$
\mathrm{P}_{2}^{1}(x y)=\mathrm{P}_{2}^{1}(x) y+\mathrm{Q}_{1}(x) \mathrm{Q}_{1}(y)+x \mathrm{P}_{2}^{1}(y) .
$$

Using $\mathbf{Q}_{1}\left(\zeta_{i}\right)=\zeta_{i-2}^{4}$, we get

$$
\mathrm{P}_{2}^{1}\left(\zeta_{i} \zeta_{j}\right)=\zeta_{i-2}^{4} \zeta_{j-2}^{4} \quad \mathrm{P}_{2}^{1}\left(\zeta_{i}^{2}\right)=\zeta_{i-2}^{8} .
$$

Formulas become more complicated for triple products, e.g.

$$
\mathrm{P}_{2}^{1}\left(\zeta_{i} \zeta_{j} \zeta_{k}\right)=\zeta_{i-2}^{4} \zeta_{j-2}^{4} \zeta_{k}+\zeta_{i-2}^{4} \zeta_{j} \zeta_{k-2}^{4}+\zeta_{i} \zeta_{j-2}^{4} \zeta_{k-2}^{4},
$$

and in general we have the following result.

Lemma 2.8. The action of $\mathrm{P}_{2}^{1}$ on $\mathfrak{T}$ is given by the formula

$$
\begin{aligned}
\mathrm{P}_{2}^{1}\left(\zeta_{i_{1}} \ldots \zeta_{i_{n}}\right) & =\sum_{1 \leq j<k \leq n} \frac{\zeta_{i_{1}} \ldots \zeta_{i_{n}}}{\zeta_{i_{j}} \zeta_{i_{k}}} \mathrm{Q}_{1}\left(\zeta_{i_{j}}\right) \mathrm{Q}_{1}\left(\zeta_{i_{k}}\right) \\
& =\sum_{1 \leq j<k \leq n} \zeta_{i_{1}} \ldots \zeta_{i_{j-1}} \zeta_{i_{j}-2}^{4} \zeta_{i_{j+1}} \ldots \zeta_{i_{k-1}} \zeta_{i_{k}-2}^{4} \zeta_{i_{k+1}} \ldots \zeta_{i_{n}}
\end{aligned}
$$


where indices are allowed to repeat.

Proof. Follows from an inductive argument on $n$, using (2.6) and the facts that $\mathrm{P}_{2}^{1}\left(\zeta_{i}\right)=0$ and $\mathrm{Q}_{1}\left(\zeta_{i}\right)=\zeta_{i-2}^{4}$.

The technique developed in this paper begins with the following observation. Consider the subalgebra

$$
\mathcal{E}:=\mathbb{F}_{2}\left[\zeta_{1}^{8}, \zeta_{2}^{4}, \zeta_{3}^{2}, \zeta_{4}^{2}, \zeta_{5}^{2}, \ldots\right] \subset \mathfrak{T}=\mathbb{F}_{2}\left[\zeta_{1}^{8}, \zeta_{2}^{4}, \zeta_{3}^{2}, \zeta_{4}, \zeta_{5}, \ldots\right]
$$

which we will call the even subalgebra of $\mathfrak{T}$, as every element in $\mathcal{E}$ has even grading. Since $\left|Q_{1}\right|=3$ and every element in $\mathcal{E}$ has even grading, $Q_{1}$ must act trivially on $\mathcal{E}$. Thus, $\mathrm{P}_{2}^{1}$ restricted to $\mathcal{E}$ follows the Leibniz rule, hence $\mathcal{M}\left(\mathcal{E}^{\otimes r}, \mathrm{P}_{2}^{1}\right)$ is an algebra. Using (2.7) and the Künneth isomorphism for a derivation, we can easily deduce the following result.

Lemma 2.9. The $\mathrm{P}_{2}^{1}$ Margolis homology of $\mathcal{E}$ is given by

$$
\mathcal{M}\left(\mathcal{E}, \mathrm{P}_{2}^{1}\right) \cong \Lambda\left(\zeta_{2}^{4}, \zeta_{3}^{4}, \zeta_{4}^{4}, \ldots\right)
$$

Moreover

$$
\mathcal{M}\left(\mathcal{E}^{\otimes r}, \mathrm{P}_{2}^{1}\right) \cong \mathcal{M}\left(\mathcal{E}, \mathrm{P}_{2}^{1}\right)^{\otimes r} \cong\left(\Lambda\left(\zeta_{2}^{4}, \zeta_{3}^{4}, \zeta_{4}^{4}, \ldots\right)\right)^{\otimes r}
$$

Now consider the quotient $\mathcal{K}:=\mathfrak{T} / / \mathcal{E} \cong \mathbb{F}_{2} \otimes_{\mathcal{E}} \mathfrak{T}$. We have an isomorphism $\mathcal{K} \cong \Lambda\left(\zeta_{4}, \zeta_{5}, \ldots\right)$, and the induced action of $\mathrm{Q}_{1}$ and $\mathrm{P}_{2}^{1}$ on $\mathcal{K}$ is trivial. For a set $A$, we let $\mathbb{F}_{2}\langle A\rangle$ denote the $\mathbb{F}_{2}$-vector space which has the generating set $A$. The algebra $\mathcal{K}$ admits a natural increasing filtration

$$
G^{p}(\mathcal{K}):=\mathbb{F}_{2}\left\langle\zeta_{i_{1}} \ldots \zeta_{i_{k}} \mid k \leq p\right\rangle
$$

induced by the length of the monomials. We call it the length filtration.

This length filtration on $\mathcal{K}$ induces an increasing filtration $\left\{G^{p}(\mathfrak{T})\right\}_{p \geq 0}$ on $\mathfrak{T}$, where $G^{p}(\mathfrak{T})$ is the pullback of $G^{p}(\mathcal{K})$ (in vector spaces) along the quotient map $\mathfrak{T} \rightarrow \mathcal{K}$

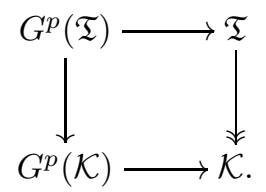

Definition 2.10. Let $I$ be a finite tuple of natural numbers, and for $I=\left\{i_{1}, \ldots, i_{n}\right\}$ let $\zeta^{I}$ denote the monomial $\zeta_{1}^{i_{1}} \ldots \zeta_{n}^{i_{n}}$. Then the length $L$ of $\zeta^{I}$ is defined by

$$
L\left(\zeta^{I}\right)=\sum_{j=1}^{|I|}\left(i_{j} \bmod 2\right) .
$$

In other words, $L\left(\zeta^{I}\right)$ counts the number of odd exponents in $\zeta^{I}$. Then $G^{p}(\mathfrak{T})$ is the span of monomials $\zeta^{I}$ of length less than or equal to $p$

$$
G^{p}(\mathfrak{T}) \cong \mathbb{F}_{2}\left\langle\zeta^{I} \mid L\left(\zeta^{I}\right) \leq p\right\rangle .
$$

The length function $L$ measures "how far" a given monomial in $\mathfrak{T}$ is from the even subalgebra $\mathcal{E}$. Since there is an $\mathbb{F}_{2}$-vector space isomorphism

$$
\mathfrak{T} \cong \mathcal{E} \otimes \mathfrak{T} / / \mathcal{E}=\mathcal{E} \otimes \mathcal{K}
$$

any monomial $m \in \mathfrak{T}$ can be uniquely written as $e \cdot k$ where $e \in \mathcal{E}$ and $k \in \mathcal{K}$. 
Example 2.11. If $m=\zeta_{3}^{4} \zeta_{5}^{5} \zeta_{8}^{3}$, then there is an unique expression $m=e \cdot k$, where $e=\zeta_{3}^{4} \zeta_{5}^{4} \zeta_{8}^{2} \in \mathcal{E}$ and $k=\zeta_{5} \zeta_{8} \in \mathcal{K}$.

The following lemma shows that the action of $Q_{1}$ and $P_{2}^{1}$ preserves the length filtration.

Lemma 2.12. Let $m \in \mathfrak{T}$ be any monomial.

(i) If $m \in \mathcal{E}$, then $\mathrm{Q}_{1}(m)=0$ and $\mathrm{P}_{2}^{1}(m) \in \mathcal{E}$.

(ii) If $m \notin \mathcal{E}$, then $\mathrm{Q}_{1}(m) \in G^{L(m)-1}(\mathfrak{T}) / G^{L(m)-2}(\mathfrak{T})$ and

$$
\mathrm{P}_{2}^{1}(m)=m_{L}+m_{L-2},
$$

where $m_{L} \in G^{L(m)}(\mathfrak{T}) / G^{L(m)-1}(\mathfrak{T})$ and $m_{L-2} \in G^{L(m)-2}(\mathfrak{T}) / G^{L(m)-3}(\mathfrak{T})$.

Proof. When $m \in \mathcal{E}, \mathbf{Q}_{1}(m)=0$ by the Leibniz rule. Using Lemma 2.8 we have $\mathrm{P}_{2}^{1}(m) \in \mathcal{E}$ and $L\left(\mathrm{P}_{2}^{1}(m)\right)=L(m)=0$.

Now assume $m \notin \mathcal{E}$, which means $m=e \cdot k$ for some $e \in \mathcal{E}$ and some $1 \neq k \in \mathcal{K}$. Note that $k$ is of the form $\zeta_{i_{1}} \ldots \zeta_{i_{n}}$, where indices do not repeat.

The action of $Q_{1}$ is given by the formula

$$
\mathrm{Q}_{1}\left(\zeta_{i_{1}} \ldots \zeta_{i_{n}}\right)=\sum_{k=1}^{n} \zeta_{i_{1}} \ldots \zeta_{i_{k-1}} \zeta_{i_{k}-2}^{4} \zeta_{i_{k+1}} \ldots \zeta_{i_{n}}
$$

where we allow repetition of indices. Since $Q_{1}$ acts trivially on $\mathcal{E}$, it follows that

$$
\mathrm{Q}_{1}(e \cdot k)=e \cdot \mathrm{Q}_{1}(k) .
$$

From the formula above we see that $\mathrm{Q}_{1}(k) \neq 0$ and $L\left(\mathrm{Q}_{1}(k)\right)=L(k)-1$. Hence,

$$
L\left(\mathrm{Q}_{1}(m)\right)=L\left(e \cdot \mathrm{Q}_{1}(k)\right)=L\left(\mathrm{Q}_{1}(k)\right)=L(k)-1=L(e \cdot k)-1=L(m)-1 .
$$

Next, note that

$$
\mathrm{P}_{2}^{1}(m)=\mathrm{P}_{2}^{1}(e) \cdot k+\mathrm{Q}_{1}(e) \cdot \mathrm{Q}_{1}(k)+e \cdot \mathrm{P}_{2}^{1}(k)=\mathrm{P}_{2}^{1}(e) \cdot k+e \cdot \mathrm{P}_{2}^{1}(k)
$$

From Lemma 2.8 , we see that $L\left(\mathrm{P}_{2}^{1}(k)\right)=L\left(\mathrm{P}_{2}^{1}(k)\right)-2$ assuming $\mathrm{P}_{2}^{1}(k) \neq 0$. Now set $m_{L}=\mathrm{P}_{2}^{1}(e) \cdot k$ and $m_{L-2}=e \cdot \mathrm{P}_{2}^{1}(k)$

Lemma 2.13. The Hopf algebra $\Lambda\left(\mathrm{Q}_{1}, \mathrm{P}_{2}^{1}\right)$ is commutative and cocommutative.

Proof. Commutativity follows from the fact that $\mathrm{P}_{2}^{1}$ and $\mathrm{Q}_{1}$ commute [AM71, Lemma 1.3(2)] (in the notation of [AM71], $\mathrm{P}_{2}^{1}=P_{2}(2)$ and $\mathrm{Q}_{1}=P_{2}(1)$ ). Cocommutativity follows from the fact that the diagram

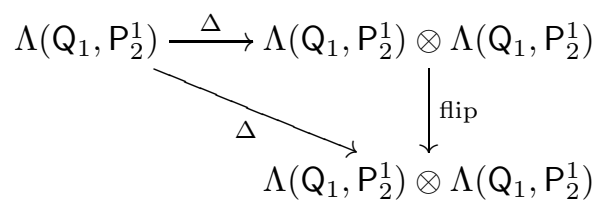

commutes, because of (1.3) and (1.4). 
If $M$ is a $\Lambda\left(\mathrm{Q}_{1}, \mathrm{P}_{2}^{1}\right)$-module then let $\mathcal{C}_{M}^{\bullet}$ denote the periodic chain complex

$$
\ldots \stackrel{\mathrm{P}_{2}^{1}}{\longrightarrow} M \stackrel{\mathrm{P}_{2}^{1}}{\longrightarrow} M \stackrel{\mathrm{P}_{2}^{1}}{\longrightarrow} \ldots .
$$

Its homology groups are isomorphic in each degree, i.e.

$$
H_{i}\left(\mathcal{C}_{M}^{\bullet}\right) \cong H_{j}\left(\mathcal{C}_{M}^{\bullet}\right)
$$

for all $i, j \in \mathbb{Z}$. We use $\mathcal{M}\left(M, \mathrm{P}_{2}^{1}\right)$ to denote this common homology group. When $M=\mathfrak{T}$, the filtration $G^{\bullet}(\mathfrak{T})$ induces a filtration on $C_{\mathfrak{T}}^{\bullet}$. By Lemma 2.12, $\mathrm{P}_{2}^{1}$ respects the length filtration. This means we have a short exact sequence of chain complexes

$$
0 \longrightarrow \bigoplus_{p \in \mathbb{Z}} G^{p-1}\left(C_{\mathfrak{T}}^{\bullet}\right) \longrightarrow \bigoplus_{p \in \mathbb{Z}} G^{p}\left(C_{\mathfrak{T}}^{\bullet} \longrightarrow \bigoplus_{p \in \mathbb{Z}} \frac{G^{p}\left(C_{\mathfrak{\mathfrak { T }}}\right)}{G^{p-1}\left(C_{\mathfrak{\mathfrak { x }}}^{\bullet}\right)} \longrightarrow 0\right.
$$

Upon taking the homology, this short exact sequence of chain complexes produces an exact couple, resulting in a spectral sequence

$$
E_{1}^{p, q}:=H^{q}\left(\frac{G^{p}\left(C_{\mathfrak{T}}^{\bullet}\right)}{G^{p-1}\left(C_{\mathfrak{T}}^{\bullet}\right)}\right) \Rightarrow H^{q}\left(C_{\mathfrak{T}}^{\bullet}\right) .
$$

We rewrite this spectral sequence as

$$
E_{1}^{p}:=\mathcal{M}\left(\frac{G^{p}(\mathfrak{T})}{G^{p-1}(\mathfrak{T})}, \mathrm{P}_{2}^{1}\right) \Rightarrow \mathcal{M}\left(t m f, \mathrm{P}_{2}^{1}\right) .
$$

and we call it the length spectral sequence.

The $E_{1}$ page of (2.14) is easy to calculate. Note that the associated graded

$$
\bigoplus_{p \geq 0} \frac{G^{p}(\mathfrak{T})}{G^{p-1}(\mathfrak{T})} \cong \mathcal{E} \otimes \mathcal{K}
$$

as an $\mathbb{F}_{2}$-algebra. The action of $\Lambda\left(\mathrm{Q}_{1}, \mathrm{P}_{2}^{1}\right)$ on $\mathcal{E} \otimes \mathcal{K}$ is defined using the Cartan formula as in the definition below.

Definition 2.15 ([Mar83], p.186). Let $\Gamma$ be any Hopf algebra. For two $\Gamma$-modules $M$ and $N$, the underlying $\mathbb{F}_{2}$ vector space of $M \otimes N$ is simply $M \otimes_{\mathbb{F}_{2}} N$, and $\Gamma$ acts via the diagonal map, i.e.

$$
a(m \otimes n)=\sum_{i} a_{i}(m) \otimes a_{i}^{\prime}(n) .
$$

where $a \in \Gamma$ and $\Delta(a)=\sum_{i} a_{i} \otimes a_{i}^{\prime}$, where $\Delta$ is the coproduct of the Hopf algebra.

Now we describe the action of $\mathrm{P}_{2}^{1}$ on a monomial $m \in \bigoplus_{p \geq-1} \frac{G^{p}(\mathfrak{T})}{G^{p-1}(\mathfrak{T})}$. Write $m=e \otimes k$ for some $e \in \mathcal{E}$ and $k \in \mathcal{K}$. By Definition 2.15

$$
\mathrm{P}_{2}^{1}(m)=\mathrm{P}_{2}^{1}(e \otimes k)=\mathrm{P}_{2}^{1}(e) \otimes k .
$$

Since the length filtration $G^{\bullet}(\mathfrak{T})$ is multiplicative, i.e.

$$
G^{p}(\mathfrak{T}) \cdot G^{p^{\prime}}(\mathfrak{T}) \subset G^{p+p^{\prime}}(\mathfrak{T})
$$

and $\mathrm{P}_{2}^{1}$ restricted to $\mathcal{E}$ follows the Leibniz rule, the $E_{1}$ page of $(2.14)$ is an $\mathbb{F}_{2}$-algebra and isomorphic to

$$
E_{1}^{*} \cong \mathcal{M}\left(\mathcal{E} \otimes \mathcal{K}, \mathrm{P}_{2}^{1}\right) \cong \mathcal{M}\left(\mathcal{E}, \mathrm{P}_{2}^{1}\right) \otimes \mathcal{K} \cong \Lambda\left(\zeta_{2}^{4}, \zeta_{3}^{4}, \ldots\right) \otimes \Lambda\left(\zeta_{4}, \zeta_{5}, \ldots\right) .
$$

In order to avoid confusion regarding the multiplicative structure of $E_{1}^{*}$, it is convenient to rename the generators. 
Notation 2.16. We set $x_{i}:=\zeta_{i+3}$ and $t_{i}:=\zeta_{i+1}^{4}$. Further, for finite subsets $I=\left\{i_{1}, \ldots, i_{n}\right\} \subset \mathbb{N}$ and $J=\left\{j_{1}, \ldots, j_{m}\right\} \subset \mathbb{N}$, we let $t_{I}$ and $x_{I}$ denote the monomials $t_{i_{1}} \ldots t_{i_{n}}$ and $x_{j_{1}} \ldots x_{j_{n}}$ respectively. We use $t_{I} x_{J}$ to denote the tensor product $t_{I} \otimes x_{J}$.

Lemma 2.12 and Lemma 2.13 imply that we have a commutative diagram of chain complexes

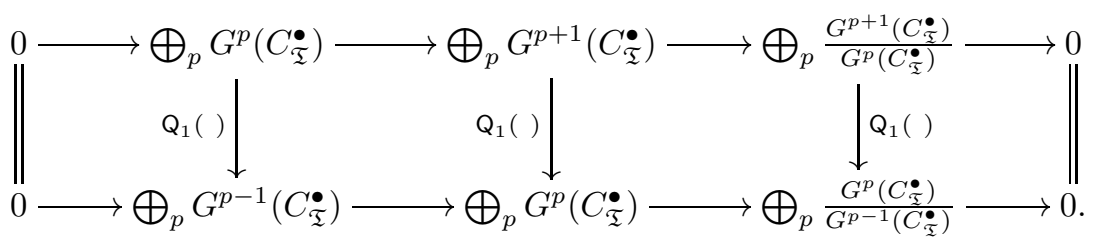

Consequently there is an action of $Q_{1}$ on each page of (2.14), which shifts the length filtration by -1 . In particular, we note $\mathrm{Q}_{1}\left(x_{i}\right)=t_{i}$ and in general

$$
\mathrm{Q}_{1}\left(t_{I} x_{J}\right)=\sum_{j \in J} t_{j} t_{I} x_{J-\{j\}}
$$

Let $m \in \mathfrak{T}$ be any monomial, $m_{L}$ and $m_{L-2}$ be as in Lemma 2.12, and let $[m]$ denote the equivalence class in the $E_{1}$ page of (2.14) represented by $m$. Lemma 2.12 implies that the $d_{1}$ differential of (2.14) is trivial,

$$
d_{2}([m])=\left[m_{L-2}\right]
$$

for the class of the monomial $m \in \mathfrak{T}$ in the $E_{1}$ page, and the spectral sequence (2.14) collapses at the $E_{3}$ page. If we write $m \in \mathfrak{T}$ as $m=e \cdot k$, where $e \in \mathcal{E}$ and $k \in \mathcal{K}$, then

$$
d_{2}([m])=\left[e \cdot \mathrm{P}_{2}^{1}(k)\right]=[e] \cdot\left[\mathrm{P}_{2}^{1}(k)\right] .
$$

This means that the $d_{2}$ differential of $(2.14)$ is $\mathcal{M}\left(\mathcal{E}, \mathrm{P}_{2}^{1}\right)$-linear. It follows from the formula of Lemma 2.8 that

$$
d_{2}\left(t_{I} x_{J}\right)=\sum_{K \in J[2]} t_{K} t_{I} x_{J-K}
$$

where $J[2]$ is the set of subsets of $J$ which contain two elements.

The formula for the $d_{2}$ differentials is intimately related to the action of $\mathrm{Q}_{1}$ on the $E_{2}$ page of (2.14). The $\Lambda\left(Q_{1}\right)$-module structure on $E_{2}^{\bullet}$ (see (2.17)) can extend it to the $\Lambda\left(\mathrm{Q}_{1}, \mathrm{P}_{2}^{1}\right)$-module structure using the algebra structure of $E_{2}^{\bullet}$ and the product formula (2.6)), along with the initial condition

$$
\mathrm{P}_{2}^{1}\left(x_{i}\right)=\mathrm{P}_{2}^{1}\left(t_{i}\right)=0 .
$$

The action of $\mathrm{P}_{2}^{1}$ that results from this procedure is

$$
\mathrm{P}_{2}^{1}\left(t_{I} x_{J}\right)=\sum_{K \in J[2]} t_{K} t_{I} x_{J-K}
$$

on the monomial basis, which can be extended to all of $E_{2}^{\bullet}$ using $\mathbb{F}_{2}$-linearity. Notice that the action we obtain through this process coincides with the formula for the $d_{2}$ differentials (2.18). 


\section{The REDUCED LENGTH}

For convenience, we denote the $E_{2}$-page of (2.14) by

$$
\mathcal{R}=\Lambda\left(t_{i}: i \geq 1\right) \otimes \Lambda\left(x_{i}: i \geq 1\right)
$$

which is an $\mathbb{F}_{2}$-algebra, as well as a $\Lambda\left(\mathrm{Q}_{1}, \mathrm{P}_{2}^{1}\right)$-module, where the actions of $\mathrm{Q}_{1}$ and $P_{2}^{1}$ are given by (2.17) and (2.20) respectively. In this section we analyze the $\Lambda\left(\mathrm{Q}_{1}, \mathrm{P}_{2}^{1}\right)$-module structure of $\mathcal{R}$, which leads us to a description of

$$
E_{\infty}^{\bullet} \cong \ldots \cong E_{3}^{\bullet} \cong H\left(E_{2}^{\bullet}, d_{2}\right) \cong \mathcal{M}\left(\mathcal{R}, \mathrm{P}_{2}^{1}\right)
$$

The main idea here is to notice that the action of $\mathrm{P}_{2}^{1}$ is linear with respect to the subalgebra

$$
\mathcal{S}:=\Lambda\left(t_{i} x_{i} \mid i \in \mathbb{N}_{+}\right) \subset \mathcal{R},
$$

which implies that $\mathcal{M}\left(\mathcal{R}, \mathrm{P}_{2}^{1}\right)$ admits an $\mathcal{S}$-module structure.

Lemma 3.1. The subalgebra $\mathcal{S} \subset \mathcal{R}$ is a trivial $\Lambda\left(\mathrm{Q}_{1}, \mathrm{P}_{2}^{1}\right)$-submodule which splits off as a $\Lambda\left(\mathrm{Q}_{1}, \mathrm{P}_{2}^{1}\right)$-module.

Proof. For any element $t_{I} x_{I} \in \mathcal{S}$, it is clear from (2.17) and (2.18) that

$$
\mathrm{Q}_{1}\left(t_{I} x_{I}\right)=0=\mathrm{P}_{2}^{1}\left(t_{I} x_{I}\right) .
$$

Thus $\mathcal{S}$ is a trivial submodule.

Now observe from (2.17) and (2.18) that none of the monomials $t_{I} x_{I} \in \mathcal{S}$ is a summand of $\mathrm{Q}_{1}\left(t_{I^{\prime}} x_{J^{\prime}}\right)$ or $\mathrm{P}_{2}^{1}\left(t_{I^{\prime}} x_{J^{\prime}}\right)$ for any choice of $I^{\prime}$ and $J^{\prime}$. Hence, $\mathcal{S}$ is a split summand.

Corollary 3.2. Every element of $\mathcal{S}$ is a nonzero cycle in the $\mathcal{M}\left(\mathcal{R}, \mathrm{P}_{2}^{1}\right)$.

Lemma 3.3. The action of $\mathrm{P}_{2}^{1}$ on $\mathcal{R}$ is $\mathcal{S}$-linear.

Proof. It is enough to show that

$$
\mathrm{P}_{2}^{1}\left(t_{i} x_{i} \cdot t_{I} x_{J}\right)=\left(t_{i} x_{i}\right) \cdot \mathrm{P}_{2}^{1}\left(t_{I} x_{J}\right) .
$$

If $i \in I$, then $t_{i} t_{I}=0$. Hence both the LHS and the RHS of (3.4) are zero.

If $i \in J$, then $x_{i} x_{J}=0$, hence LHS of (3.4) is zero. On the other hand,

$$
\begin{aligned}
R H S & =t_{i} x_{i} \cdot \sum_{K \in J[2]} t_{K} t_{I} x_{J-K} \\
& =\sum_{i \in K \in J[2]} t_{i} t_{K} t_{I} x_{i} x_{J-K}+t_{i} \cdot\left(\sum_{i \notin K \in J[2]} t_{i} t_{I} x_{i} x_{J-K}\right)=0,
\end{aligned}
$$

as $t_{i} t_{K}=0$ when $i \in K$ and $x_{i} x_{J-K}=0$ when $i \notin K$. 
Now consider the case when $i \notin I \cup J$. Let $I^{\prime}=I \cup\{i\}$ and $J^{\prime}=J \cup\{i\}$. Then,

$$
\begin{aligned}
\mathrm{P}_{2}^{1}\left(t_{i} x_{i} \cdot t_{I} x_{J}\right)=\mathrm{P}_{2}^{1}\left(t_{I^{\prime}} x_{J^{\prime}}\right) & =\sum_{K \in J^{\prime}[2]} t_{K} t_{I^{\prime}} x_{J^{\prime}-K} \\
& =\sum_{i \in K \in J^{\prime}[2]} t_{K} t_{I^{\prime}} x_{J^{\prime}-K}+\sum_{i \notin K \in J^{\prime}[2]} t_{K} t_{I^{\prime}} x_{J^{\prime}-K} \\
& =\sum_{i \notin K \in J^{\prime}[2]} t_{K} t_{I^{\prime}} x_{J^{\prime}-K} \\
& =t_{i} x_{i} \cdot \sum_{K \in J[2]} t_{K} t_{I} x_{J-K} \\
& =t_{i} x_{i} \cdot \mathrm{P}_{2}^{1}\left(t_{I} x_{J}\right) .
\end{aligned}
$$

Remark 3.5. While the $E_{2}$ page of (2.14) admits an $\mathbb{F}_{2}$-algebra structure, the $E_{3}$ page does not admit any multiplicative structure. This is because the $d_{2}$ differentials do not follow Leibniz rule and the product of $d_{2}$ cycles may not be a cycle. For example, $x_{i}$ for all $i \in \mathbb{N}_{+}$, is a $d_{2}$-cycle, whereas $x_{i} x_{j}$ for $i \neq j$ supports a differential $d_{2}\left(x_{i} x_{j}\right)=t_{i} t_{j}$ by (2.18). Even if $\alpha, \beta$ and $\alpha \cdot \beta$ are $\mathrm{P}_{2}^{1}$ cycles it is unclear that the pairing $[\alpha] \cdot[\beta]=[\alpha \cdot \beta]$ is well-defined in the $E_{3}$ page.

Corollary 3.6. $\mathcal{M}\left(\mathcal{R}, \mathrm{P}_{2}^{1}\right)$ is a module over the ring $\mathcal{S}$.

Proof. By Lemma 3.3, there exists a pairing $\mu: \mathcal{S} \otimes \mathcal{R} \rightarrow \mathcal{R}$ such that the diagram

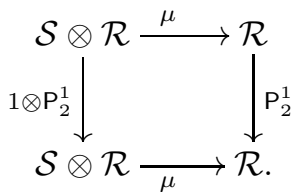

commutes. It follows that $\mathcal{M}\left(\mathcal{R}, \mathrm{P}_{2}^{1}\right)$ is an $\mathcal{S}$ module.

As a result, we only need to understand the action of $\mathrm{P}_{2}^{1}$ on the generators of $\mathcal{R}$ when viewed as an $\mathcal{S}$-module. In order to approach this problem we introduce the notion of reduced length.

Definition 3.7. For any monomial $t_{I} x_{J} \in \mathcal{R}$ the reduced length $\ell$ is

$$
\ell\left(t_{I} x_{J}\right)=|J-I|=\left|J \cap I^{c}\right|=|J|-|J \cap I|,
$$

where $I^{c}$ denotes the complement of $I$.

Note that the length of $t_{I} x_{J} \in \mathcal{R}$ is given by the formula $L\left(t_{I} x_{J}\right)=|J|$; in other words, it is counting the number of factors of $x_{J}$. Whereas, $\ell\left(t_{I} x_{J}\right)$ counts only those factors $x_{j}$ in $x_{J}$ for which $t_{j}$ is not a factor of $t_{I}$. For example,

$$
\begin{gathered}
\ell\left(x_{1}\right)=\ell\left(t_{1} x_{1} x_{2}\right)=\ell\left(t_{1} t_{2} x_{1} x_{2} x_{3}\right)=\ell\left(t_{1} t_{2} t_{3} x_{4}\right)=1 \\
\ell\left(x_{1} x_{2}\right)=\ell\left(t_{1} x_{1} x_{2} x_{3}\right)=\ell\left(t_{1} t_{2} t_{3} t_{4} x_{5} x_{6}\right)=2 .
\end{gathered}
$$

Remark 3.8. The reduced length function $\ell$ measures "how far" a given monomial in $\mathcal{R}$ is from the subalgebra $\mathcal{S}$. 
For each $i \in \mathbb{N}_{+}$, let $M_{i}:=\Lambda\left(\mathrm{Q}_{1}\right)\left\{x_{i}\right\} \subset \mathcal{R}$ denote the $\Lambda\left(\mathrm{Q}_{1}, \mathrm{P}_{2}^{1}\right)$-submodule isomorphic to $\Lambda\left(\mathrm{Q}_{1}\right)$ and generated by $x_{i}$. For an indexing set $K \underset{\text { finite }}{\subset} \mathbb{N}_{+}$, let

$$
M_{K}:=\bigotimes_{j \in K} M_{j} \subset \mathcal{R}
$$

with the convention that $M_{\emptyset}:=\mathbb{F}_{2}$. If the indexing set is $[n]=\{1, \ldots, n\} \subset \mathbb{N}_{+}$, then we write $M_{[n]}$ to denote $M_{\{1, \ldots, n\}}$.

In Figure 1, Figure 2 and Figure 3 we present $M_{i}, M_{\{1,2\}}$ and $M_{\{1,2,3\}}$ respectively as a $\Lambda\left(\mathrm{Q}_{1}, \mathrm{P}_{2}^{1}\right)$-module. In these figures the blue curved lines depict the action of $\mathrm{Q}_{1}$ and red boxed lines depict the action of $\mathrm{P}_{2}^{1}$.

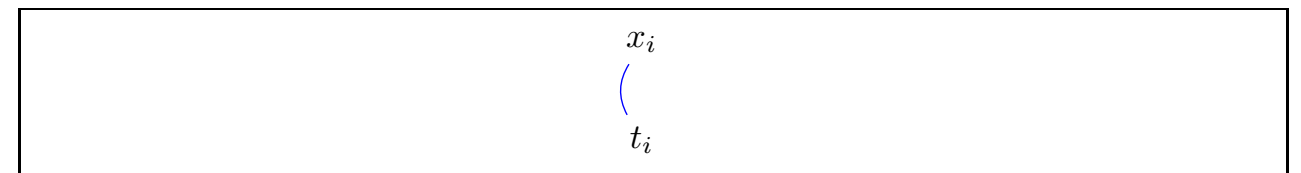

Figure 1: $M_{i}$ as a module over $\Lambda\left(\mathrm{Q}_{1}, \mathrm{P}_{2}^{1}\right)$

Note that the set $\mathcal{W}:=\left\{t_{I} x_{J} \in \mathcal{R} \mid I \cap J=\emptyset\right\}$ forms a generating set for $\mathcal{R}$ as an $\mathcal{S}$-module as any monomial $t_{I} x_{J} \in \mathcal{R}$ can be uniquely written as a product of an element of $\mathcal{W}$ and a monomial in $\mathcal{S}$ :

$$
t_{I} x_{J}=t_{I \cap J} x_{I \cap J} \cdot t_{I-(I \cap J)} x_{J-(I \cap J)} .
$$

For any finite subset $K \subset \mathbb{N}_{+}$,

$$
\mathcal{W}_{K}:=\left\{t_{I} x_{J} \mid I \cup J=K, I \cap J=\emptyset\right\} \subset \mathcal{W}
$$

forms an $\mathbb{F}_{2}$-basis for $M_{K}$, i.e. $\mathbb{F}_{2}\left\langle\mathcal{W}_{K}\right\rangle=M_{K}$. Since

$$
\mathcal{W}=\bigsqcup_{\substack{\text { finite }_{\perp} \\ \subset}} \mathcal{W}_{K}
$$

and $\mathbb{F}_{2}\left\langle\mathcal{W}_{K}\right\rangle=M_{K}$ is closed under the action of $\mathrm{Q}_{1}$ and $\mathrm{P}_{2}^{1}$ (these actions preserve the total indexing set $K$, by (2.17) and (2.18)), we learn that

$$
\mathcal{R} / / \mathcal{S} \cong \mathbb{F}_{2} \otimes_{\mathcal{S}} \mathcal{R} \cong \mathcal{R} \otimes_{\mathcal{S}} \mathbb{F}_{2} \cong \bigoplus_{K} M_{K}
$$

is an isomorphism of $\Lambda\left(\mathrm{Q}_{1}, \mathrm{P}_{2}^{1}\right)$-modules. Consequently, we have the following lemma.

Lemma 3.9. Let $\mathcal{S}_{K} \subset \mathcal{S}$ denote the subalgebra $\Lambda\left(t_{I} x_{I} \mid I \subset \mathbb{N}_{+}-K\right)$. There is a $\Lambda\left(\mathrm{Q}_{1}, \mathrm{P}_{2}^{1}\right)$-module isomorphism

$$
\bigoplus_{\substack{K \subset \mathcal{c}_{\text {finite }} \\ \mathbb{N}_{+}}} \mathcal{S}_{K} \otimes M_{K} \cong \mathcal{R} .
$$

Proof. Consider the $\mathbb{F}_{2}$-vector space isomorphism

$$
\iota: \mathcal{R} \longrightarrow \underset{\substack{K \\ \text { finite }}}{\bigoplus_{\mathbb{N}_{+}}} \mathcal{S}_{K} \otimes M_{K}
$$




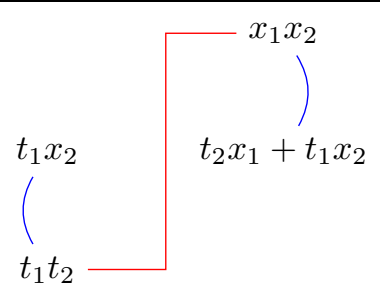

Figure 2: $M_{[2]}$ as a module over $\Lambda\left(\mathrm{Q}_{1}, \mathrm{P}_{2}^{1}\right)$, where $[2]=\{1,2\}$

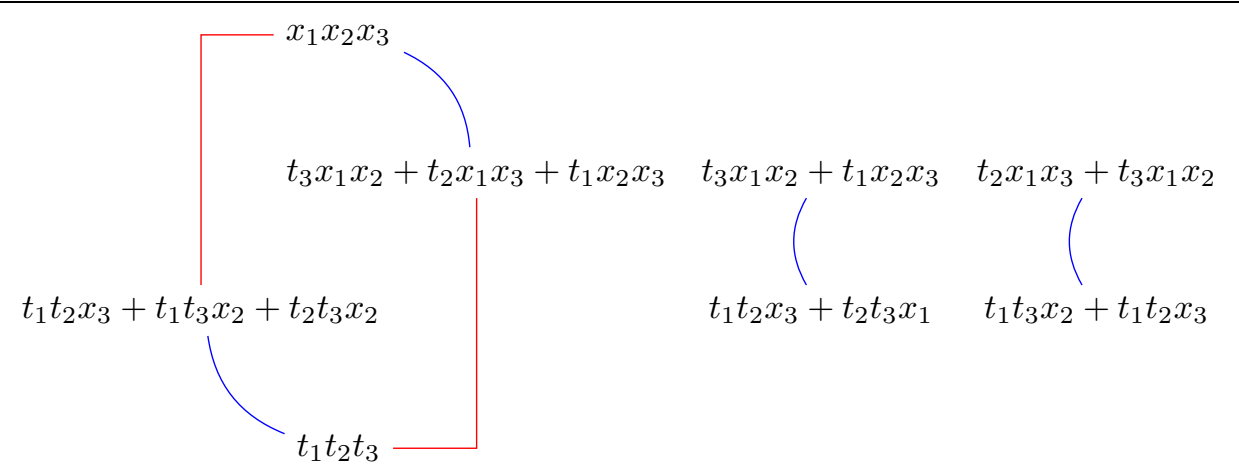

Figure 3: $M_{[3]}$ as a module over $\Lambda\left(\mathrm{Q}_{1}, \mathrm{P}_{2}^{1}\right)$, where $[3]=\{1,2,3\}$

which sends

$$
t_{I} x_{J} \mapsto t_{I \cap J} x_{I \cap J} \otimes t_{I-(I \cap J)} x_{J-(I \cap J)} \in \mathcal{S}_{K} \otimes M_{K},
$$

where $K=I \cup J-I \cap J$. The map $\iota^{-1}$ sends

$$
t_{B} x_{B} \otimes t_{K-A} x_{A} \mapsto t_{B \cup(K-A)} \cdot x_{B \cup A},
$$

where $A \subset K$. This map is also a $\Lambda\left(\mathrm{Q}_{1}, \mathrm{P}_{2}^{1}\right)$-module isomorphism as $\mathcal{S}_{K} \subset \mathcal{S}$ is a trivial $\Lambda\left(\mathrm{Q}_{1}, \mathrm{P}_{2}^{1}\right)$-module by Lemma 3.1.

Hence we can reduce the problem of computing $\mathcal{M}\left(\mathcal{R}, \mathrm{P}_{2}^{1}\right)$ to computing $\mathcal{M}\left(M_{K}, \mathrm{P}_{2}^{1}\right)$ for various finite subsets $K$ of $\mathbb{N}_{+}$. Thus, we first need to understand the structure of $M_{K}$ as a $\Lambda\left(\mathrm{Q}_{1}, \mathrm{P}_{2}^{1}\right)$-module.

Remark 3.10. Let $[n]$ denote the indexing set $\{1, \ldots, n\} \subset \mathbb{N}_{+}$. If $|K|=n$, then there exists the unique order preserving bijection

$$
\iota:[n] \longrightarrow K
$$

and it induces an isomorphism $\iota: M_{[n]} \cong M_{K}$. Thus it is enough to understand $\Lambda\left(\mathrm{Q}_{1}, \mathrm{P}_{2}^{1}\right)$-module structure of $M_{[n]}$ for all $n \in \mathbb{N}_{+}$.

As depicted in Figure $3, M_{[3]}$ splits as a $\Lambda\left(\mathrm{Q}_{1}, \mathrm{P}_{2}^{1}\right)$-module

$$
\begin{aligned}
& M_{[3]} \cong \quad \Lambda\left(\mathrm{Q}_{1}, \mathrm{P}_{2}^{1}\right)\left\{x_{1} x_{2} x_{3}\right\} \\
& \oplus \Lambda\left(\mathrm{Q}_{1}\right)\left\{t_{3} x_{1} x_{2}+t_{1} x_{2} x_{3}\right\} \\
& \oplus \Lambda\left(Q_{1}\right)\left\{t_{2} x_{1} x_{3}+t_{3} x_{1} x_{2}\right\}
\end{aligned}
$$

as summand of a free $\Lambda\left(Q_{1}, P_{2}^{1}\right)$-module and two copies of $\Lambda\left(Q_{1}\right)$. 
Remark 3.12. The splitting of (3.11) is a consequence of Lemma 2.13. Since $\Lambda\left(\mathrm{Q}_{1}, \mathrm{P}_{2}^{1}\right)$ is cocommutative, for any $\Lambda\left(\mathrm{Q}_{1}, \mathrm{P}_{2}^{1}\right)$-module $M$ and $\sigma \in \mathbb{F}_{2}\left[\Sigma_{n}\right]$, the induced map

$$
\sigma: M^{\otimes n} \longrightarrow M^{\otimes n}
$$

is a map of $\Lambda\left(\mathrm{Q}_{1}, \mathrm{P}_{2}^{1}\right)$-modules. Note that in the group ring $\mathbb{F}_{2}\left[\Sigma_{3}\right]$, the identity element can be written as a sum of idempotent elements

$$
\mathbb{1}=e+f_{1}+f_{2} .
$$

For example, one can choose $e=\mathbb{1}+\left(\begin{array}{lll}1 & 2 & 3\end{array}\right)+\left(\begin{array}{lll}1 & 2 & 3\end{array}\right), f_{1}=\mathbb{1}+\left(\begin{array}{ll}1 & 2\end{array}\right)+\left(\begin{array}{ll}1 & 3\end{array}\right)+\left(\begin{array}{lll}1 & 3 & 2\end{array}\right)$ and $f_{2}=\mathbb{1}+\left(\begin{array}{ll}1 & 2\end{array}\right)+\left(\begin{array}{ll}1 & 3\end{array}\right)+\left(\begin{array}{lll}1 & 2 & 3\end{array}\right)$. Then we have

$$
M^{\otimes 3} \cong e\left(M^{\otimes 3}\right) \oplus f_{1}\left(M^{\otimes 3}\right) \oplus f_{2}\left(M^{\otimes 3}\right) .
$$

When $M \cong \Lambda\left(\mathrm{Q}_{1}\right)$, we get the decomposition of of (3.11).

The splitting of (3.11), along with the following fact about finite dimensional Hopf algebras, is the key to understanding the structure of $M_{K}$.

Theorem 3.13 ([NZ89]). If $\mathcal{H}$ is a finite dimensional connected Hopf algebra over a field $\mathbb{F}$, then for any $\mathcal{H}$-module $M, \mathcal{H} \otimes M$ is a free $\mathcal{H}$-module.

Let us denote by $A$ the $\Lambda\left(\mathrm{Q}_{1}, \mathrm{P}_{2}^{1}\right)$-module isomorphic to $\Lambda\left(\mathrm{Q}_{1}\right)$ and let $B:=A \otimes A$.

Then using (3.11) and Theorem 3.13, we notice that

$$
\begin{aligned}
M_{[3]} \cong B \otimes A \cong\{\text { Free }\} \oplus A^{\oplus 2} \\
M_{[4]} \cong\{\text { Free }\} \oplus B^{\oplus 2} \\
M_{[5]} \cong\{\text { Free }\} \oplus A^{\oplus 4}
\end{aligned}
$$

where $\{$ Free $\}$ denotes a free $\Lambda\left(\mathrm{Q}_{1}, \mathrm{P}_{2}^{1}\right)$ module. This iterative process can be continued as described in Lemma 3.14 below. We use $A\{y\}$, resp. $B\{y\}$, to specify that $y$ generates $A$, resp. $B$, as a $\Lambda\left(\mathrm{Q}_{1}, \mathrm{P}_{2}^{1}\right)$ module. For example, $M_{i} \cong A\left\{x_{i}\right\}$.

Lemma 3.14. As a $\Lambda\left(\mathrm{Q}_{1}, \mathrm{P}_{2}^{1}\right)$-module,

$$
M_{[2 r+1]} \cong\{\text { Free }\} \oplus\left(\bigoplus_{i=1}^{2^{r}} A\left\{h_{2 r+1, i}\right\}\right),
$$

where $\ell\left(h_{2 r+1, i}\right)=r+1$.

As a $\Lambda\left(\mathrm{Q}_{1}, \mathrm{P}_{2}^{1}\right)$-module,

$$
M_{[2 r]} \cong\{\text { Free }\} \oplus \bigoplus_{i=1}^{2^{r-1}} B\left\{h_{2 r, i}\right\},
$$

where $\ell\left(h_{2 r, i}\right)=r+1$.

Proof. Our proof is by induction on $r$. From Figure 1, Figure 2 and Figure 3, the claim is true for $k=1,2,3$. Note that

$h_{1,1}=x_{1} \quad h_{2,1}=x_{1} x_{2} \quad h_{3,1}=\left(t_{3} x_{1}+x_{3} t_{1}\right) x_{2} \quad h_{3,2}=\left(t_{2} x_{3}+t_{3} x_{2}\right) x_{1}$

Now assume that the result is true for $2 r-1$, i.e.

$$
M_{[2 r-1]} \cong\{\text { Free }\} \oplus \bigoplus_{1 \leq i \leq 2^{r-1}} A\left\{h_{2 r-1, i}\right\}
$$


where $\ell\left(h_{2 r-1, i}\right)=r$ and $\{$ Free $\}$ is a free $\Lambda\left(\mathrm{Q}_{1}, \mathrm{P}_{2}^{1}\right)$-module. It follows that

$$
M_{[2 r]} \cong M_{[2 r-1]} \otimes M_{2 r} \cong\left(\{\text { Free }\} \otimes A\left\{x_{2 r}\right\}\right) \oplus \bigoplus_{1 \leq i \leq 2^{r-1}} B\left\{h_{2 r-1, i} \cdot x_{2 r}\right\} .
$$

By Theorem 3.13, the first summand is, again, a free module. Set

$$
h_{2 r, i}=h_{2 r-1, i} \cdot x_{2 r}
$$

and notice $\ell\left(h_{2 r-1, i} x_{2 r}\right)=\ell\left(h_{2 r-1, i}\right)+\ell\left(x_{2 r}\right)=r+1$.

To complete the inductive argument, observe

$$
\begin{aligned}
M_{[2 r+1]} \cong M_{[2 r-1]} \otimes B\left\{x_{2 r} x_{2 r+1}\right\} \\
\cong\left(\{\text { Free }\} \oplus \bigoplus_{1 \leq i \leq 2^{r-1}} A\left\{h_{2 r-1, i}\right\}\right) \otimes B\left\{x_{2 r} x_{2 r+1}\right\} \\
\cong\left\{\text { Free } \oplus \oplus \bigoplus_{1 \leq i \leq 2^{r-1}}\left(A\left\{h_{2 r+1,2 i-1}\right\} \oplus A\left\{h_{2 r+1,2 i}\right\}\right),\right.
\end{aligned}
$$

where one can define the generators $h_{2 r-1, j}$ from Figure 3 by replacing $x_{1}, x_{2}, x_{3}$ with $h_{2 r-1, i}, x_{2 r}$ and $x_{2 r+1}$ respectively. More specifically, one can define

$$
\begin{gathered}
h_{2 r+1,2 i-1}=\mathrm{Q}_{1}\left(h_{2 r-1, i} \cdot x_{2 r+1}\right) \cdot x_{2 r} \\
h_{2 r+1,2 i}=h_{2 r-1, i} \cdot \mathrm{Q}_{1}\left(x_{2 r} x_{2 r+1}\right) .
\end{gathered}
$$

It is easy to check that $\ell\left(h_{2 r+1, j}\right)=r+1$.

Following the proof of Lemma 3.14, we can provide an explicit basis of $\mathcal{M}\left(M_{K}, \mathrm{P}_{2}^{1}\right)$. By Remark 3.10 it suffices to provide a basis for $\mathcal{M}\left(M_{[n]}, \mathrm{P}_{2}^{1}\right)$ for all $n \geq 1$. We do so inductively (see Definition 3.16), however we must treat the odd and the even case separately, essentially because of Lemma 3.14. Since $A$ is a trivial $\Lambda\left(\mathrm{P}_{2}^{1}\right)$-module, $\mathcal{M}\left(A, \mathrm{P}_{2}^{1}\right) \cong A$, and we get

$$
\mathcal{M}\left(M_{[2 r+1]}, \mathrm{P}_{2}^{1}\right) \cong \mathcal{M}\left(\bigoplus_{i=1}^{2^{r}} A\left\{h_{2 r+1, i}\right\}, \mathrm{P}_{2}^{1}\right) \cong \bigoplus_{i=1}^{2^{r}} A\left\{h_{2 r+1, i}\right\}
$$

Thus the collection

$$
\left\{h_{2 r+1, i}: 1 \leq i \leq 2^{r}\right\} \cup\left\{\mathrm{Q}_{1}\left(h_{2 r+1, i}\right): 1 \leq i \leq 2^{r}\right\}
$$

is an $\mathbb{F}_{2}$-basis of $\mathcal{M}\left(M_{[2 r+1]}, \mathrm{P}_{2}^{1}\right)$. When $n$ is even, say $n=2 r$, then

$$
\mathcal{M}\left(M_{[2 r]}, \mathrm{P}_{2}^{1}\right) \cong \bigoplus_{i=1}^{2^{r-1}} \mathcal{M}\left(B\left\{h_{2 r, i}\right\}, \mathrm{P}_{2}^{1}\right) .
$$

Now note that, if $B\{x \otimes y\}=A\{x\} \otimes A\{y\}$ (where $x$ and $y$ are generators), then

$$
\left\{\mathrm{Q}_{1}(x) \otimes y, x \otimes \mathrm{Q}_{1}(y)\right\}
$$

is an $\mathbb{F}_{2}$-basis of $\mathcal{M}\left(B\{x \otimes y\}, \mathrm{P}_{2}^{1}\right)$. Using the fact that

$$
h_{2 r, i}=h_{2 r-1, i} \cdot x_{2 r},
$$

we get Corollary 3.15 and Definition 3.16 thereafter. 
Corollary 3.15. Let $\mathcal{M}\left(M_{K}, \mathrm{P}_{2}^{1}\right)_{l}=\left\{x \in \mathcal{M}\left(M_{K}, \mathrm{P}_{2}^{1}\right) \mid \ell(x)=l\right\}$.

If $|K|=2 r+1$, then

$$
\operatorname{dim} \mathcal{M}\left(M_{K}, \mathrm{P}_{2}^{1}\right)_{l}=\left\{\begin{array}{cc}
2^{r}, & \text { if } l=r, r+1 \\
0, & \text { otherwise }
\end{array}\right.
$$

If $|K|=2 r$, then

$$
\operatorname{dim} \mathcal{M}\left(M_{K}, \mathrm{P}_{2}^{1}\right)_{l}=\left\{\begin{array}{cc}
2^{r}, & \text { if } l=r \\
0, & \text { otherwise }
\end{array}\right.
$$

Proof. Lemma 3.14 implies

$$
M_{[2 r+1]} \cong\{\text { Free }\} \oplus \bigoplus_{1 \leq i \leq 2^{r}} A\left\{h_{2 r+1, i}\right\}
$$

where $\ell\left(h_{2 r+1, i}\right)=r+1$. By Lemma 2.12 we have $\ell\left(\mathrm{Q}_{1}\left(h_{2 r+1, i}\right)\right)=r$. Thus $\left\{h_{2 r+1, i}\right\}$ is the basis for $\mathcal{M}\left(M_{[2 r+1]}, \mathrm{P}_{2}^{1}\right)_{r+1}$ and $\left\{\mathrm{Q}_{1}\left(h_{2 r+1, i}\right)\right\}$ is the basis for $\mathcal{M}\left(M_{[2 r+1]}, \mathrm{P}_{2}^{1}\right)_{r}$. Applying Remark 3.10 we deduce the statement about dimension for any $M_{K}$ with $|K|=2 r+1$.

For the even case we have from Lemma 3.14

$$
M_{[2 r]} \cong\{\text { Free }\} \oplus \bigoplus_{1 \leq i \leq 2^{r-1}} B\left\{h_{2 r, i}\right\}
$$

where $\ell\left(h_{2 r, i}\right)=r+1$. Then for each $i, \mathcal{M}\left(B\left\{h_{2 r, i}\right\}, \mathrm{P}_{2}^{1}\right)=\mathcal{M}\left(B\left\{h_{2 r, i}\right\}, \mathrm{P}_{2}^{1}\right)_{r}$ is an $\mathbb{F}_{2}$ vector space of dimension 2 generated by $\left\{h_{2 r-1, i} \cdot t_{2 r}, \mathrm{Q}_{1}\left(h_{2 r-1, i}\right) \cdot x_{2 r}\right\}$.

Definition 3.16. We define the basis $\mathcal{B}_{[n], l}$ of $\mathcal{M}\left(M_{[n]}, \mathrm{P}_{2}^{1}\right)_{l}$ for $0 \leq l \leq n$ inductively starting with $\mathcal{B}_{[1], 0}=\left\{t_{1}\right\}$ and $\mathcal{B}_{[1], 1}=\left\{x_{1}\right\}$. Suppose we have defined

$$
\mathcal{B}_{[2 r-1], l}:=\left\{\begin{array}{cl}
\left\{h_{2 r-1,1}, \ldots, h_{2 r-1,2^{r-1}}\right\} & \text { if } l=r \\
\left\{\mathrm{Q}_{1}\left(h_{2 r-1,1}\right), \ldots, \mathrm{Q}_{1}\left(h_{2 r-1,2^{r-1}}\right)\right\} & \text { if } l=r-1 \\
\emptyset & \text { otherwise. }
\end{array}\right.
$$

Then define:

$\mathcal{B}_{[2 r], r}:=\left\{h_{2 r-1,1} \cdot t_{2 r}, \ldots, h_{2 r-1,2^{r-2}} \cdot t_{2 r}\right\} \cup\left\{\mathbf{Q}_{1}\left(h_{2 r-1,1}\right) \cdot x_{2 r}, \ldots, \mathbf{Q}_{1}\left(h_{2 r-1,2^{r-2}}\right) \cdot x_{2 r}\right\}$ and set $\mathcal{B}_{[2 r], l}:=\emptyset$ if $l \neq r$.

Now define $h_{2 r+1,2 i-1}=\mathrm{Q}_{1}\left(h_{2 r-1, i}\right) \cdot\left(x_{2 r+1} \cdot x_{2 r}\right)$ and $h_{2 r+1,2 i}=h_{2 r-1, i} \cdot \mathrm{Q}_{1}\left(x_{2 r} x_{2 r+1}\right)$ and set

$$
\mathcal{B}_{[2 r+1], l}:=\left\{\begin{array}{cl}
\left\{h_{2 r+1,1}, \ldots, h_{2 r+1,2^{r-2}}\right\} & \text { if } l=r+1 \\
\left\{Q_{1}\left(h_{2 r+1,1}\right), \ldots, Q_{1}\left(h_{2 r+1,2^{r-2}}\right)\right\} & \text { if } l=r \\
\emptyset & \text { otherwise. }
\end{array}\right.
$$

We let $\mathcal{B}_{[n]}$ denote the union $\bigcup_{l} \mathcal{B}_{[n], l}$. Let $\mathcal{B}_{K}$ denote the image of the $\mathcal{B}_{[n]}$ under the isomorphism $\iota: M_{[n]} \rightarrow M_{K}$ of Remark 3.10.

Example 3.17 (Examples of $\mathcal{B}_{K}$ ). We explicitly identify $\mathcal{B}_{[n]}$ using Definition 3.16 for $n \leq 4$, and for $n=1,2,3$ we can compare to Figures 1,2 and 3 , to see that $\mathcal{B}_{[n]}$ is indeed the basis for $\mathcal{M}\left(M_{[n]}, \mathrm{P}_{2}^{1}\right)$.

- $\mathcal{B}_{[1]}=\left\{t_{1}, x_{1}\right\}$,

- $\mathcal{B}_{[2]}=\left\{t_{1} x_{2}, t_{2} x_{1}\right\}$, 
- $\mathcal{B}_{[3]}=\left\{t_{1} t_{2} x_{3}+t_{1} t_{3} x_{2}, t_{1} t_{2} x_{3}+t_{2} t_{3} x_{1}\right\} \cup\left\{t_{3} x_{1} x_{2}+t_{2} x_{1} x_{3}, t_{3} x_{1} x_{2}+t_{1} x_{2} x_{3}\right\}$, and,

- $\mathcal{B}_{[4]}=\left\{t_{1} t_{2} x_{3} x_{4}+t_{1} t_{3} x_{2} x_{4}, t_{1} t_{2} x_{3} x_{4}+t_{2} t_{3} x_{1} x_{4}, t_{3} t_{4} x_{1} x_{2}+t_{2} t_{4} x_{1} x_{3}, t_{3} t_{4} x_{1} x_{2}+\right.$ $\left.t_{1} t_{4} x_{2} x_{3}\right\}$.

Let $P_{K}:=\mathbb{F}_{2}\left\langle\mathcal{B}_{K}\right\rangle \subset M_{K}$. By construction, $P_{K}$ is a trivial split $\Lambda\left(\mathrm{P}_{2}^{1}\right)$-submodule of $M_{K}$. The splitting of $P_{K}$ follows from the fact that its complement is free and the fact that $\Lambda\left(\mathrm{P}_{2}^{1}\right)$, like any other finite dimensional connected Hopf algebra, is self-injective [Mar83, Ch. 12, §2].

Theorem 3.18. Let $K$ be a finite subset of $\mathbb{N}_{+}$. Let

$$
\mathcal{S B}_{K}:=\left\{t_{I} x_{I} \cdot b \mid I \cap K=\emptyset \text { and } b \in \mathcal{B}_{K}\right\} \subset \mathcal{R} .
$$

Then

$$
\mathcal{B}:=\bigsqcup_{\substack{K \\ \text { finite }}} \mathcal{\mathbb { N } _ { + }}
$$

forms a basis of the $\mathbb{F}_{2}$-vector space $\mathcal{M}\left(\right.$ tmf, $\left.\mathrm{P}_{2}^{1}\right)$ and

$$
\mathcal{M}\left(t m f, \mathrm{P}_{2}^{1}\right) \cong \bigoplus_{\substack{\mathrm{f}_{\text {finte }} \mathbb{N}_{+} \\ \mathbb{N}_{2}}} \mathbb{F}_{2}\left\langle\mathcal{S} \mathcal{B}_{K}\right\rangle \cong \bigoplus_{K_{\text {finite }} \subset \mathbb{N}_{+}} \mathcal{S}_{K} \otimes \mathcal{M}\left(M_{K}, \mathrm{P}_{2}^{1}\right) .
$$

is an isomorphism of $\mathbb{F}_{2}$-vector spaces.

Proof. By Lemma 3.9, we have a $\Lambda\left(\mathrm{Q}_{1}, \mathrm{P}_{2}^{1}\right)$ module isomorphism

$$
\mathcal{R} \cong \underset{\substack { K \\
\begin{subarray}{c}{\mathrm{finite}^{\subset} \mathbb{N}_{+}{ K \\
\begin{subarray} { c } { \mathrm { finite } ^ { \subset } \mathbb { N } _ { + } } }\end{subarray}}{ } \mathcal{S}_{K} \otimes M_{K}
$$

Therefore, the linearity of the action of $\mathrm{P}_{2}^{1}$ (see Corollary 3.6) with respect to elements in $\mathcal{S}$ gives us

$$
\begin{aligned}
\mathcal{M}\left(t m f, \mathrm{P}_{2}^{1}\right) & \cong \mathcal{M}\left(\mathcal{R}, \mathrm{P}_{2}^{1}\right) \\
& \cong \mathcal{M}\left(\bigoplus_{K{ }_{\text {finite }} \mathbb{N}_{+}} \mathcal{S}_{K} \otimes M_{K}, \mathrm{P}_{2}^{1}\right) \\
& \cong \bigoplus_{K_{\text {finite }} \mathbb{N}_{+}} \mathcal{S}_{K} \otimes \mathcal{M}\left(M_{K}, \mathrm{P}_{2}^{1}\right) \\
& \cong \bigoplus_{\substack{K_{\text {finite }} \\
\mathbb{N}_{+}}} \mathcal{S}_{K} \otimes P_{K} \\
& \cong \bigoplus_{\prod_{\text {finite }} \subset \mathbb{N}_{+}} \mathbb{F}_{2}\left\langle\mathcal{S} \mathcal{B}_{K}\right\rangle .
\end{aligned}
$$

Remark 3.19. Let $e$ denote the exchange map $e: \mathcal{R} \rightarrow \mathcal{R}$ which sends

$$
e: t_{I} x_{J} \mapsto t_{J} x_{I} .
$$

It seems to be the case that $[m] \in \mathcal{M}\left(t m f, \mathrm{P}_{2}^{1}\right)$ if and only if $[e(m)] \in \mathcal{M}\left(t m f, \mathrm{P}_{2}^{1}\right)$. The source of such symmetry is unclear to the authors, although it might be related to Spanier-Whitehead duality. 
Finally, we would like to say a word about the module structure of $\mathcal{M}\left(t m f, \mathrm{P}_{2}^{1}\right)$ over $\mathcal{S}$. Note that the collection of elements

$$
\mathcal{B}_{\mathcal{S}}:=\left\{t_{I} x_{I} \mid I \underset{\text { finite }}{\subset} \mathbb{N}_{+}\right\}
$$

forms an $\mathbb{F}_{2}$-basis of $\mathcal{S}$. The $\mathcal{S}$-module structure on $\mathcal{M}\left(t m f, \mathrm{P}_{2}^{1}\right)$ is extended from a pairing at the level of bases

$$
\begin{aligned}
& \mathcal{B}_{\mathcal{S}} \otimes \mathcal{S B}_{K} \stackrel{\mu}{\rightarrow} \mathcal{S B}_{K} \\
& s \otimes\left(s^{\prime} \cdot b\right) \mapsto\left\{\begin{array}{l}
\left(s \cdot s^{\prime}\right) \cdot b, \text { if } I \cap K=\emptyset \\
0, \text { if } I \cap K \neq \emptyset .
\end{array}\right.
\end{aligned}
$$

Remark 3.20. Recall that $H_{*}(t m f)$ was described in terms of $\zeta_{i}$. We can convert an element of the Margolis homology expressed in terms of $t_{i}$ and $x_{i}$ back to an expression involving $\zeta_{i}$ using the identifications of Notation 2.16. For example,

$$
t_{4} t_{9} x_{2} x_{6}+t_{2} t_{9} x_{4} x_{6}
$$

can be identified with the class represented by element $\zeta_{5}^{5} \zeta_{10}^{4} \zeta_{9}+\zeta_{3}^{4} \zeta_{10}^{4} \zeta_{7} \zeta_{9} \in \mathfrak{T}$.

$$
\text { 4. } P_{2}^{1} \text { Margolis homology of } t m f^{\wedge r} \text { AND } B\left(\mathbb{Z} / 2^{\times n}\right)_{+}
$$

4.1. $\mathrm{P}_{2}^{1}$ Margolis homology of $t m f^{\wedge r}$. Note that

$$
H_{*}\left(t m f^{\wedge r}\right) \cong H_{*}(t m f)^{\otimes r} \cong \mathfrak{T}^{\otimes r} .
$$

We first extend the notion of length to $\mathfrak{T}^{\otimes r}$. For a monomial $\zeta^{I_{1}}|\ldots| \zeta^{I_{r}}$ for $\zeta^{I_{i}} \in$ $\mathfrak{T}^{\otimes r}$, which is a tensor product of monomials in $\mathfrak{T}$, we define

$$
L\left(\zeta^{I_{1}}|\ldots| \zeta^{I_{r}}\right)=L\left(\zeta^{I_{1}}\right)+\cdots+L\left(\zeta^{I_{r}}\right) .
$$

We define the even subalgebra $\mathbb{E}_{r}$ of $\mathfrak{T}^{\otimes r}$ as the span of those monomials in $\mathfrak{T}^{\otimes r}$ whose lengths are zero. Observe that,

$$
\mathbb{E}_{r} \cong \mathcal{E}^{\otimes r}
$$

Notion of length leads to an increasing filtration on $\mathfrak{T}^{\otimes r}$, call it the length filtration, by setting

$$
G^{p}\left(\mathfrak{T}^{\otimes r}\right)=\left\{\left(\zeta^{I_{1}}|\ldots| \zeta^{I_{r}}\right) \mid L\left(\zeta^{I_{1}}|\ldots| \zeta^{I_{r}}\right) \leq p\right\}
$$

Let $\mathbb{K}_{r}=\mathcal{K}^{\otimes r}$, where $\mathcal{K}$ is as defined in Section 2. Just like in the case $r=1$, we get a length spectral sequence and its $E_{1}$ page is

$$
E_{1}^{\bullet} \cong \mathcal{M}\left(\mathbb{E}_{r}, \mathrm{P}_{2}^{1}\right) \otimes \mathbb{K}_{r} \Rightarrow \mathcal{M}\left(t m f^{\wedge r}, \mathrm{P}_{2}^{1}\right) .
$$

Since action of $\mathrm{P}_{2}^{1}$ follows the Leibniz rule when restricted to $\mathcal{E}$, we get

$$
\mathcal{M}\left(\mathbb{E}_{r}, \mathrm{P}_{2}^{1}\right) \cong \mathcal{M}\left(\mathcal{E}, \mathrm{P}_{2}^{1}\right)^{\otimes r}
$$

Notation 4.2. For shorthand, we denote $x_{i, j}=(\underbrace{1|\ldots| 1}_{j-1}\left|\zeta_{i+3}\right| \underbrace{1|\ldots| 1}_{r-j})$ and $t_{i, j}=$ $(\underbrace{1|\ldots| 1}_{j-1}\left|\zeta_{i+1}^{4}\right| \underbrace{|\ldots| 1}_{r-j})$. With this notation we have

$$
\mathrm{Q}_{1}\left(x_{i, j}\right)=t_{i, j}
$$


Using Notation 4.2, we see that the $E_{1}$ page of the length spectral sequence (4.1), as an algebra, is isomorphic to

$$
\mathcal{R}_{r}:=\Lambda\left(t_{i, j}: i \in \mathbb{N}-\{0\}, 1 \leq j \leq r\right) \otimes \Lambda\left(x_{i, j}: i \in \mathbb{N}-\{0\}, 1 \leq j \leq r\right) .
$$

It is easy to see that the map induced by the reindexing map

$$
\iota:(i, j) \mapsto r(i-1)+j,
$$

produces a (non-canonical) isomorphism of algebras between $\mathcal{R}_{r}$ (the $E_{2}$ page of (4.1)) and $\mathcal{R}$ (the $E_{2}$ page of (2.14)), after forgetting the internal grading. This is also an isomorphism of $\Lambda\left(\mathrm{Q}_{1}, \mathrm{P}_{2}^{1}\right)$-modules. Thus we have an isomorphism

$$
\iota_{*}: \mathcal{M}\left(t m f, \mathrm{P}_{2}^{1}\right) \stackrel{\cong}{\longrightarrow} \mathcal{M}\left(t m f^{\wedge r}, \mathrm{P}_{2}^{1}\right)
$$

induced by the $\iota$. Therefore, Theorem 3.18 essentially gives a complete calculation of $\mathcal{M}\left(t m f^{\wedge r}, \mathrm{P}_{2}^{1}\right)$.

Example 4.3. For example, let us assume $r=3$. Then the element $t_{2} t_{4} x_{6} x_{9}+$ $t_{2} t_{6} x_{4} x_{9} \in \mathcal{M}\left(t m f, \mathrm{P}_{2}^{1}\right)$ (see Example 3.17) corresponds to the element

$$
t_{1,2} t_{2,1} x_{2,3} x_{3,3}+t_{1,2} t_{2,3} x_{2,1} x_{3,3} \in \mathcal{M}\left(t m f^{\wedge 3}, \mathrm{P}_{2}^{1}\right)
$$

under the bijection obtained from the above reindexing. When expressed in terms of $\zeta_{i}$ s (see Notation 4.2), the same element can be expressed as

$$
\zeta_{3}^{4}\left|\zeta_{2}^{4}\right| \zeta_{5} \zeta_{6}\left|1+\zeta_{5}\right| \zeta_{2}^{4}\left|\zeta_{3}^{4} \zeta_{6}\right| 1
$$

Remark 4.4 ( $\mathrm{P}_{2}^{1}$ Margolis homology of Brown-Gitler spectra). It is well-known that

$$
H_{*}(t m f) \cong \bigoplus_{i \geq 0} H_{*}\left(\Sigma^{8 i} b o_{i}\right)
$$

where $b o_{i}$ are certain Brown-Gitler spectra associated with bo. In [Mah81] Mahowald defined a multiplicative weight function, which is given by $w\left(\zeta_{i}\right)=2^{i-1}$. $H_{*}\left(\Sigma^{8 i} b o_{i}\right)$ is the summand of $H_{*}(t m f)$ which consists of elements of Mahowald weight exactly equal to $8 i$. We assign Mahowald weight of $t_{i, j}$ and $x_{i, j}$ as

$$
w\left(t_{i, j}\right)=w\left(x_{i, j}\right)=2^{i+1} .
$$

It follows that the Margolis homology $\mathcal{M}\left(b o_{q_{1}} \wedge \cdots \wedge b o_{q_{r}}, \mathrm{P}_{2}^{1}\right)$ is a summand of $\mathcal{M}\left(t m f^{\wedge r}, \mathrm{P}_{2}^{1}\right)$. It consists of all polynomials of $\mathcal{M}\left(t m f^{\wedge r}, \mathrm{P}_{2}^{1}\right)$ expressed in terms of $x_{i, j}$ and $t_{i, j}$ such that $w\left(x_{i, j}\right)=w\left(t_{i, j}\right)=4 q_{j}$.

Remark 4.5. While it is true that $\mathcal{R}_{r} \cong \mathcal{R}^{\otimes r}$, as an $\mathbb{F}_{2}$-algebra as well as an $\Lambda\left(\mathrm{Q}_{1}, \mathrm{P}_{2}^{1}\right)$-module, it is not useful for the purposes of calculating $\mathcal{M}\left(\mathcal{R}_{r}, \mathrm{P}_{2}^{1}\right)$. This is because $P_{2}^{1}$ does not obey Leibniz rule and

$$
\mathcal{M}\left(\mathcal{R}_{r}, \mathrm{P}_{2}^{1}\right) \neq \mathcal{M}\left(\mathcal{R}, \mathrm{P}_{2}^{1}\right)^{\otimes r} .
$$

However we overcome this difficulty by producing an $\Lambda\left(\mathrm{Q}_{1}, \mathrm{P}_{2}^{1}\right)$-module isomorphism $\iota_{*}$ at the expense of forgetting the internal grading. 
4.2. $\mathrm{P}_{2}^{1}$ Margolis homology of $\left(B \mathbb{Z} / 2^{\times k}\right)_{+}$. The space $B \mathbb{Z} / 2$ is also known as $\mathbb{R P}^{\infty}$, the real infinite-dimensional projective space. It is well-known that

$$
H^{*}\left((B \mathbb{Z} / 2)_{+}, \mathbb{F}_{2}\right) \cong \mathbb{F}_{2}[x]
$$

and therefore

$$
H^{*}\left(\left(B \mathbb{Z} / 2^{\times k}\right)_{+}, \mathbb{F}_{2}\right) \cong \mathbb{F}_{2}\left[x_{1}, \ldots x_{k}\right]
$$

It can be seen that $\mathrm{P}_{2}^{1}\left(x_{i}\right)=0$ and $\mathrm{Q}_{1}\left(x_{i}\right)=x_{i}^{4}$. We again define the length function on the monomials in the usual way

$$
L\left(x_{1}^{i_{1}} \ldots x_{k}^{i_{k}}\right)=\left(i_{1} \bmod 2\right)+\cdots+\left(i_{k} \bmod 2\right) .
$$

The even complex $\mathcal{E}$, which is the span of elements of length zero, is isomorphic to

$$
\mathcal{E}=\mathbb{F}_{2}\left[x_{1}^{2}, \ldots, x_{k}^{2}\right]
$$

It can be seen that $\mathrm{P}_{2}^{1}\left(x_{i}^{2}\right)=x_{i}^{8}$. Now observe that $\mathrm{Q}_{1}$ acts trivially on $\mathcal{E}$, hence $\mathrm{P}_{2}^{1}$ acts as a derivation and, therefore,

$$
\mathcal{M}\left(\mathcal{E}, \mathrm{P}_{2}^{1}\right) \cong \Lambda\left(x_{1}^{4}, \ldots, x_{k}^{4}\right) .
$$

Now the length function gives us an increasing length filtration

$$
G^{p}\left(\mathbb{F}_{2}\left[x_{1}, \ldots, x_{k}\right]\right)=\mathbb{F}_{2}\left\langle x_{1}^{i_{1}} \ldots x_{k}^{i_{k}}: L\left(x_{1}^{i_{1}} \ldots x_{k}^{i_{k}}\right) \leq p\right\rangle .
$$

This results in a length spectral sequence which only has $d_{0}$ and $d_{2}$ differentials. If we denote $x_{i}^{4}$ by $t_{i}$ for convenience, we can see that the length spectral sequence

$$
E_{1}^{\bullet}=\Lambda\left(t_{1}, \ldots, t_{k}\right) \otimes \Lambda\left(x_{1}, \ldots, x_{k}\right) \Rightarrow \mathcal{M}\left(\left(B \mathbb{Z} / 2^{\times k}\right)_{+}, \mathrm{P}_{2}^{1}\right)
$$

is a sub spectral sequence of (2.14) and is, in fact, isomorphic to it when $k=\infty$. Thus, when $k$ is finite, we can recover a complete description of $\mathcal{M}\left(\left(B \mathbb{Z} / 2^{\times k}\right)_{+}, \mathrm{P}_{2}^{1}\right)$ from Theorem 3.18. More precisely, we obtain

$$
\mathcal{M}\left(\left(B \mathbb{Z} / 2^{\times k}\right)_{+}, \mathrm{P}_{2}^{1}\right) \cong \bigoplus_{K \subset[k]} S_{K} \otimes \mathcal{M}\left(M_{K}, \mathrm{P}_{2}^{1}\right)
$$

where $S_{K}=\Lambda\left(t_{i} x_{i} \mid i \in[k]-K\right) . \mathcal{M}\left(\left(B \mathbb{Z} / 2^{\times k}\right)_{+}, \mathrm{P}_{2}^{1}\right)$ is a module over $\mathcal{S}_{[k]}$.

Example 4.6. $\mathcal{M}\left(\mathbb{R P}_{+}^{\infty}, \mathrm{P}_{2}^{1}\right) \cong \mathbb{F}_{2}\left\langle x_{1}, t_{1}, t_{1} x_{1}\right\rangle$, where the internal degrees of $x_{1}$ and $t_{1}$ are 1 and 4 respectively and $\mathcal{S}_{[1]}=\Lambda\left(t_{1} x_{1}\right)$. Similarly,

$$
\begin{aligned}
\mathcal{M}\left(\left(\mathbb{R} \mathbb{P}^{\infty} \times \mathbb{R P}^{\infty}\right)_{+}, \mathrm{P}_{2}^{1}\right) \cong \mathbb{F}_{2}\left\langle x_{1}, x_{2}, t_{1}, t_{2}, t_{1} x_{1}, t_{2} x_{2},\right. \\
\left.t_{1} x_{2}, t_{2} x_{1}, t_{1} x_{1} x_{2}, t_{2} x_{2} x_{1}, t_{1} t_{2} x_{2}, t_{1} t_{2} x_{1}\right\rangle
\end{aligned}
$$

where the internal degrees of $x_{i}$ and $t_{i}$ are 1 and 4 respectively. Here $\mathcal{S}_{[2]}=$ $\Lambda\left(t_{1} x_{1}, t_{2} x_{2}\right)$. If we denote

$$
H^{*}\left(\left(\mathbb{R} \mathbb{P}^{\infty} \times \mathbb{R P}^{\infty}\right)_{+}\right) \cong \mathbb{F}_{2}[y, z]
$$

where $|y|=|z|=1$, then one may choose $x_{1}=[x], x_{2}=[y], t_{1}=\left[x^{4}\right]$ and $t_{2}=\left[y^{4}\right]$. 


\section{REFERENCES}

[Ada74] J. F. Adams. Stable homotopy and generalised homology. Chicago Lectures in Mathematics. University of Chicago Press, Chicago, IL, 1974.

[AM71] J. F. Adams and H. R. Margolis. Modules over the Steenrod algebra. Topology, 10:271$282,1971$.

[Bau08] T. Bauer. Computation of the homotopy of the spectrum tmf. In Groups, homotopy and configuration spaces, volume 13 of Geom. Topol. Monogr., pages 11-40. Geom. Topol. Publ., Coventry, 2008.

$\left[\mathrm{BBB}^{+} \mathrm{a}\right]$ Agnes Beaudry, Mark Behrens, Prasit Bhattacharya, Dominic Culver, and Zhouli $\mathrm{Xu}$. On the $E_{2}$-term of the bo-Adams spectral sequence. Available at https://arxiv.org/abs/1702.00230.

$\left[\mathrm{BBB}^{+} \mathrm{b}\right]$ Agnes Beaudry, Mark Behrens, Prasit Bhattacharya, Dominic Culver, and Zhouli Xu. On the $t m f$-resolution of $\widetilde{\mathcal{Z}}$. In preparation.

[BEa] Prasit Bhattacharya and Philip Egger. A class of 2-local finite spectra which admit $v_{2}^{1}$-self-map. Available at https://arxiv.org/abs/1608.06250.

[BEb] Prasit Bhattacharya and Philip Egger. Towards the $K(2)$-local homotopy groups of $Z$. Available at https://arxiv.org/abs/1706.06170.

[BG73] Edgar H. Brown, Jr. and Samuel Gitler. A spectrum whose cohomology is a certain cyclic module over the Steenrod algebra. Topology, 12:283-295, 1973.

[BG18] Irina Bobkova and Paul G. Goerss. Topological resolutions in $K(2)$-local homotopy theory at the prime 2. Journal of Topology, 11(4):917-956, 2018.

[Cul] D. Culver. On $B P\langle 2\rangle$-cooperations. Available at https://arxiv.org/abs/1708.03001.

[DFHH14] Christopher L. Douglas, John Francis, André G. Henriques, and Michael A. Hill, editors. Topological modular forms, volume 201 of Mathematical Surveys and Monographs. American Mathematical Society, Providence, RI, 2014.

[Goe86] Paul G. Goerss. Unstable projectives and stable Ext: with applications. Proc. London Math. Soc. (3), 53(3):539-561, 1986.

[Goe10] P. G. Goerss. Topological modular forms [after Hopkins, Miller and Lurie]. Astérisque, (332):Exp. No. 1005, viii, 221-255, 2010. Séminaire Bourbaki. Volume 2008/2009. Exposés $997-1011$.

[HK00] David J. Hunter and Nicholas J. Kuhn. Characterizations of spectra with $U$-injective cohomology which satisfy the Brown-Gitler property. Trans. Amer. Math. Soc., 352(3):1171-1190, 2000.

[HM14] Michael J. Hopkins and Mark Mahowald. From elliptic curves to homotopy theory. In Topological modular forms, volume 201 of Math. Surveys Monogr., pages 261-285. Amer. Math. Soc., Providence, RI, 2014.

[HM16] Rune Haugseng and Haynes Miller. On a spectral sequence for the cohomology of infinite loop spaces. Algebr. Geom. Topol., 16(5):2911-2947, 2016.

[KM13] Nicholas Kuhn and Jason McCarty. The mod 2 homology of infinite loopspaces. Algebr. Geom. Topol., 13(2):687-745, 2013.

[LM87] Wolfgang Lellmann and Mark Mahowald. The bo-Adams spectral sequence. Trans. Amer. Math. Soc., 300(2):593-623, 1987.

[Mah81] Mark Mahowald. bo-resolutions. Pacific J. Math., 92(2):365-383, 1981.

[Mar83] H. R. Margolis. Spectra and the Steenrod algebra, volume 29 of North-Holland Mathematical Library. North-Holland Publishing Co., Amsterdam, 1983. Modules over the Steenrod algebra and the stable homotopy category.

[Mat16] Akhil Mathew. The homology of tmf. Homology Homotopy Appl., 18(2):1-29, 2016.

[Mil58] John Milnor. The Steenrod algebra and its dual. Ann. of Math. (2), 67:150-171, 1958.

[MT68] Robert E. Mosher and Martin C. Tangora. Cohomology operations and applications in homotopy theory. Harper \& Row, Publishers, New York-London, 1968.

[NZ89] Warren D. Nichols and M. Bettina Zoeller. A Hopf algebra freeness theorem. Amer. J. Math., 111(2):381-385, 1989.

[Pea14] Paul Thomas Pearson. The connective real $K$-theory of Brown-Gitler spectra. Algebr. Geom. Topol., 14(1):597-625, 2014.

[Sch94] Lionel Schwartz. Unstable modules over the Steenrod algebra and Sullivan's fixed point set conjecture. Chicago Lectures in Mathematics. University of Chicago Press, Chicago, IL, 1994. 
Department of Mathematics, University of Virginia, Kerchoff hall, Charlottesville, VA 22904

E-mail address: pb9wh@virginia.edu

Department of Mathematics, Texas A\&M University, College Station, TX 77843

E-mail address: ibobkova@math.tamu.edu

E-mail address: bt3hy@virginia.edu 